\title{
Natural products from traditional Chinese medicine for the prevention and treatment of heart failure: progress and perspectives
}

\author{
Linhao $\mathrm{Xu}^{1,2, \dagger}$, Liuying $\mathrm{Chen}^{3, \dagger}$, Gaoyang $\mathrm{Gu}^{3}$, Yi Wang ${ }^{4}$, Yizhou $\mathrm{Xu}^{1, *}$, Yigang Zhong ${ }^{1, *}$ \\ ${ }^{1}$ Department of Cardiology, Affiliated Hangzhou First People's Hospital, Zhejiang University School of Medicine, 310006 Hangzhou, Zhejiang, China \\ ${ }^{2}$ Translational Medicine Research Center, Affiliated Hangzhou First People's Hospital, Zhejiang University School of Medicine, 310006 Hangzhou, \\ Zhejiang, China \\ ${ }^{3}$ Zhejiang Chinese Medical University, 310053 Hangzhou, Zhejiang, China \\ ${ }^{4}$ Pharmaceutical Informatics Institute, College of Pharmaceutical Sciences, Zhejiang University, 310009 Hangzhou, Zhejiang, China \\ *Correspondence: xyzhzsyxnk@163.com (Yizhou Xu); zhongyigangly@163.com (Yigang Zhong) \\ $\dagger$ These authors contributed equally. \\ Academic Editors: Brian Tomlinson and Takatoshi Kasai \\ Submitted: 29 October 2021 Revised: 19 December 2021 Accepted: 20 December 2021 Published: 12 February 2022
}

\begin{abstract}
Heart failure (HF) is the end stage of several cardiovascular diseases with high mortality worldwide; however, current chemical drugs have not beneficial effect on reducing its mortality rate. Due to its properties of multiple targets components with multiple targets, natural products derived from traditional Chinese medicine (TCM) have exerts unique effects on the amelioration of the clinical symptoms of HF, yet, TCM is not widely used in the clinic since the potential therapeutic targets have not been fully investigated. Therefore, in this review, we briefly summarized the pathophysiological mechanism of HF and reviewed the published clinical evaluations of TCM and natural products from Chinese herbs to treat HF. Then, the therapeutic potential and the underlying mechanisms by which the natural products from Chinese herb exert their protective effects were further summarized. We concluded from this review that natural products from Chinese herbs have been shown to be more effective in treating HF by targeting multiple signaling pathways, including anticardiac hypertrophy, antifibrotic, anti-inflammatory, antioxidative and antiapoptotic activities. However, the major limitations of these compounds is that there are a lack of large scale, multicenter, randomized and controlled clinical trials for their use in treatment of HF, and the toxic effects of natural products from Chinese herbs also needed further investigation. Despite these limitations, further clinical trials and experimental studies will provide a better understanding of the mechanism of natural products from Chinese herbs and promote their wide use to treat HF.
\end{abstract}

Keywords: Natural products; Chinese herb; Heart failure; Cardiac hypertrophy; Inflammation; fibrosis; Oxidative stress; Myocardial apoptosis

\section{Introduction}

Heart failure (HF) is a complicated syndrome in which ventricle filling or ejection is impaired due to structural or functional cardiac disorders [1]. Globally, there are more than 37.7 million individuals with $\mathrm{HF}$, and almost $50 \%$ of HF patients die within 5 years after diagnosis; thus, the mortality rate of HF exceeds that of many cancers [2]. From a population-based study reported in 2021, the agestandardized prevalence and incidence of HF were $1.10 \%$ (an estimated 15 million HF patients) in China [3]. According to treatment guidelines, the main drugs used to treat HF in the clinic are angiotensin receptor blockers (ARBs) and mineralocorticoid receptor antagonists (MRAs). Although there has been a significant improvement in the number of hospital admissions, the 5-year mortality has not been reduced [4]. Indeed, there are some novel drugs used to treat HF in clinical trials, such as vericiguat (an oral soluble guanylate cyclase stimulator) [5], finerenone (a thirdgeneration mineralocorticoid receptor antagonist) $[6,7]$ and ferric carboxymaltose [8], however, not all of them have an apparent effect on the risk of cardiovascular death. According to one Phase IIb trial, finerenone had a beneficial effect on reducing HF mortality, however, whether the high occurrence of malignant arrhythmia and hyperkalemia caused by finerenonen still needs to be further verified [7]. Moreover, prolonged use of the above mentioned chemical drugs may result in severe side effects, such as electrolyte depletion and hypotension [9]. Therefore, natural products from Chinese herbs have been considered an alternative therapeutic strategy for the treatment of HF that is less expensive and associated with fewer side effects.

Chinese herbs have been used as complementary therapy to treat HF for a long history [10]. Traditional Chinese medicine (TCM) combined with Western medicine treatment has been shown to improve the quality of life of HF patients [11]. In China, there are many patent Chinese drugs (where the active ingredients were extracted from these herbs and made into the form of capsules, pills or injections by modern therapeutic techniques) and Chinese formulae (several herbs in different amounts are prescribed together by physicians and boiled and concentrated using a rotary 
evaporator according to the Standard Operation Procedure of the Chinese Pharmacopoeia) used to treat patients with HF; however, their mechanisms of action, complex pharmacokinetics and therapeutic targets have not been fully investigated. Therefore, TCM is often criticized or even rejected by Western scientists. In this review, we will summarize the pathogenesis of HF and clinically evaluate common Chinese formulae and patent Chinese drugs. Furthermore, putative molecular mechanism of these natural products of traditional Chinese medicine will also be reviewed with respect to current research surrounding their use in treating HF.

\section{Pathophysiology of HF}

HF is characterized by a decrease in cardiac output. According to the guidelines of American College of Cardiology Foundation (ACCF)/American Heart Association (AHA), HF with reduced ejection fraction (HFrEF) is defined as an ejection fraction $\leq 40 \%$, whereas HF with preserved ejection fraction (HFpEF) is defined as an ejection fraction $\geq 50 \%$ and $\mathrm{HF}$ with mid-range ejection fraction (HFmrEF) is defined as an ejection fraction between $41 \%$ and $49 \%$ [12]. Although the diagnosis of HFrEF, HFmrEF, and HFpEF is different, they can all lead to heart dysfunction by causing structural changes to the myocardium and activating multiple molecular signaling pathway-related processes, such as cardiomyocyte hypertrophy, endothelial dysfunction, oxidative stress, interstitial fibrosis and cardiac inflammation $[13,14]$. However, some of the mechanisms of HF development remain unclear, as few therapeutic approaches can significantly decrease the mortality of HF patients in the clinic [1]. Therefore, an improved investigation of the pathophysiological mechanisms of HF will be beneficial to evaluate and manage HF patients and provide novel therapeutic strategies to improve clinical outcomes.

\subsection{Cardiomyocyte hypertrophy}

During HF, the heart usually increases in size, which results in pathological cardiac hypertrophy. Pathological hypertrophy is characterized by contractile dysfunction and causes cardiac structural remodeling [15]. According to a previous study, calcium abnormalities and changes in gene expression play important roles in the development of cardiac hypertrophy [16] (Fig. 1).

Calcium homeostasis imbalance has been observed in $\mathrm{HF}$ and plays a major role in its progression [17]. There are two major receptors in the sarcoplasmic reticulum (SR) membrane, type 2 ryanodine receptor (RyR2) and sarcoendoplasmic reticulum calcium ATPase (SERCA), both of are involved in regulating $\mathrm{Ca}^{2+}$ homeostasis. In HF patients, it was reported that the expression and activity of SERCA2a is reduced which could reduce the amount of $\mathrm{Ca}^{2+}$ pumped back into the SR $[18,19]$. On the other hand, phosphorylation of RyR2 is enhanced in the failing human heart, sug- gesting that $\mathrm{Ca}^{2+}$ leakage from the SR is accelerated in $\mathrm{HF}$ patients and can result in contractile dysfunction [20]. Although the precise mechanism of RyR2 phosphorylation is being still debated, it has been reported that RyR2 phosphorylation is enhanced by protein kinase A (PKA) and calcium/calmodulin-dependent protein kinase II (CaMKII) [21]. Overexpression of CaMKII causes cardiac hypertrophy that rapidly transitions into HF [22], while CaMKII-KO mice are resistant to HF [23]. Therefore, specific CaMKII inhibition is a promising drug strategy for future studies. Finally, $\mathrm{Ca}^{2+}$ enters cardiomyocytes via L-type calcium channels (LTCCs) located within t-tubules; however, in the failing heart, the density of LTCCs is reduced [24]. Moreover, $\mathrm{Na}^{+} / \mathrm{Ca}^{2+}$ exchanger (NCX) activity is enhanced, which pumps $\mathrm{Ca}^{2+}$ out of the cell [25].

In addition to calcium abnormalities, the altered gene expression also contributes to the progression of cardiac hypertrophy [26]. For instance, some ligands, such as angiotensin (Ang) II, endothelin (ET)-1 and noradrenaline (NA), are closely associated with the progress of pathological cardiac hypertrophy. These ligands can bind with Gprotein-coupled receptors (GPCRs) to activate downstream signaling molecules, including phospholipase C (PLC), Protein kinase $\mathrm{C}(\mathrm{PKC})$ and mitogen-activated protein kinases (MAPKs), and then induce the expression of certain hypertrophic genes, such as atrial natriuretic peptide (ANP), B-type natriuretic peptide (BNP) and $\beta$-myosin heavy chain ( $\beta$-MHC) [27]. According to a previous study, Ang II is known to contribute to the pathogenesis of HF, and its expression was found to be increased in HF patients [28].

\subsection{Oxidative stress}

Oxidative stress, defined as the excessive generation of reactive oxygen species (ROS), has been shown to play a critical role in the pathophysiology of HF [29,30]. Normally, ROS are constantly formed in cells and removed by the antioxidant defense system; however, this balance is disturbed in HF. First, antioxidant capacity is impaired in HF; for example, the activities of superoxide dismutase (SOD) and other catalases are decreased [29]. In addition, ROS generation is enhanced in HF due to increased activity of certain enzymes, including NADPH oxidase (NOX), xanthine oxidase (XO), and uncoupled nitric oxide synthases (NOS) [31]. Therefore, we believe that oxidative stress in HF is not only induced by the enhancement of ROS generation but also by the impairment of the antioxidant defense within the heart (Fig. 1).

When excessive ROS are produced, myocardial cell injury is induced via multiple mechanisms (Fig. 1). First, ROS can impair the contractile machinery of cardiomyocytes by modifying the proteins that bind to multiple ion channels, such as calcium, potassium, and sodium channels [32]. Second, ROS can lead to contractile dysfunction by reducing myofilament calcium sensitivity and inhibiting the activity of SERCA [32]. Third, increased ROS produc- 


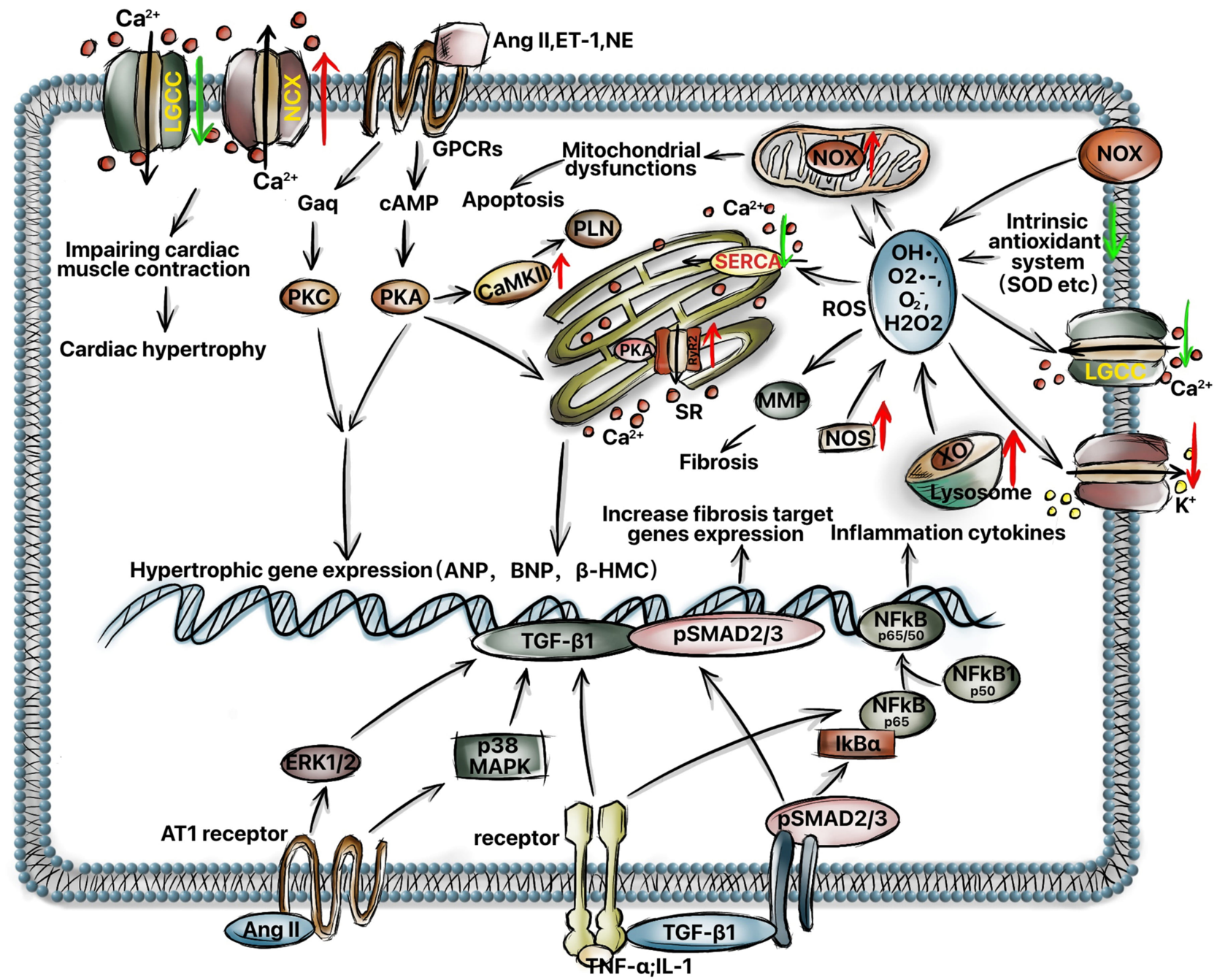

Fig. 1. A schematic of the major signaling pathways involved in cardiac hypertrophy, impaired antioxidant systems and fibrosis progression in the failing heart. First, calcium homeostasis imbalance is induced by multiple channels. The release of $\mathrm{Ca}^{2+}$ from the sarcoplasmic reticulum (SR) is accelerated by inactivation of sarcoendoplasmic reticulum calcium ATPase (SERCA2a) and the phosphorylation of type 2 ryanodine receptors (RyR2). The density of L-type $\mathrm{Ca}^{2+}$ channels (LTCCs) is reduced to decrease $\mathrm{Ca}^{2+}$ entry into the cytoplasm. The activity of $\mathrm{Na}^{+} / \mathrm{Ca}^{2+}$ exchanger (NCX) is enhanced, which pumps $\mathrm{Ca}^{2+}$ out of the cell. On the other hand, increased angiotensin II (Ang II), endothelin (ET)-1 and noradrenaline (NA) binding to G-protein-coupled receptors (GPCRs) induces the expression of hypertrophic genes, such as atrial natriuretic peptide (ANP), B-type natriuretic peptide (BNP) and $\beta$-myosin heavy chain $(\beta$-MHC). Second, reactive oxygen species (ROS) generation is also enhanced in HF due to impaired antioxidant capacity [superoxide dismutase (SOD)] and the increased activity of certain enzymes, such as NADPH oxidase (NOX), xanthine oxidase (XO), and uncoupled nitric oxide synthases (NOS). Finally, several signaling pathways are involved in fibrosis progression. Transforming growth factor $\beta$ - 1 (TGF $\beta$-1) and certain proinflammatory cytokines, such as tumor necrosis factor- $\alpha$ (TNF- $\alpha$ ), interleukin-1 (IL-1), IL-6, and IL-18, induce ECM protein gene expression and regulate fibrous tissue deposition.

tion inversely results in further mitochondrial and energy metabolism dysfunction [29]. Fourth, ROS induce cardiac fibroblast proliferation, resulting in extracellular remodeling. Finally, prolonged excessive ROS production could cause DNA damage and lead to cell damage and death.

\subsection{Myocardial fibrosis}

Cardiac fibrosis is characterized by the accumulation of extracellular matrix (ECM) in the myocardium and is an integral aspect of most pathological cardiac conditions, including HF [33]. In the healthy adult heart, cardiomyocytes are supported by ECM which is synthesized in cardiac fibroblasts. According to a previous study, the concentration of myocardial collagen increases by as much as 3- to 6-fold in HF patients [34]. Although the pathogenesis of cardiac fibrosis in HF has not been fully classified, there are two main related theories. One is that fibrotic changes are due to an accumulation of reactive collagen fibers in the inter- 
stitium and perivascular regions [35]. On the other hand, myocyte loss is suspected to stimulate replacement fibrosis to replace dead tissue. Finally, cardiac systolic and diastolic function are affected by collagen accumulation. A wide range of molecular signals are involved in this process (Fig. 1).

Transforming growth factor $\beta-1$ (TGF $\beta-1)$ is one of the most important growth factors in the development of cardiac fibrosis, and its expression has been found to be increased in HF patients [36]. Stimulation of TGF $\beta$-1 induces the transformation of fibroblasts to myofibroblasts and enhances ECM production [37], while TGF $\beta$-1 inhibition reverses these fibrotic effects [38]. When the TGF $\beta-1$ pathway is activated, it can induce ECM protein and gene expression and regulate fibrous tissue deposition via the Smad2/3 signaling pathway [39]. In addition to inducing ECM protein synthesis, TGF $\beta-1$ can also reduce collagen degradation by activating tissue inhibitors of metalloproteinases (TIMPs) [40]. Furthermore, proinflammatory cytokines are believed to play a physiological or pathological role in the development and progression of HF [41]. Increased circulating levels of tumor necrosis factor- $\alpha$ (TNF$\alpha$ ), interleukin-1 (IL-1), IL-6, and IL-18 have been reported in patients with chronic HF $[42,43]$. These cytokines can stimulate increases in cardiac fibroblast migration and enhance fibroblast sphingosine kinase activity [44]. According to a previous study, overexpression of TNF- $\alpha$ in cardiomyocytes can result in $\mathrm{HF}$, which is associated with increased collagen synthesis [45].

\subsection{Inflammation}

In HF, the innate and adaptive immune systems in the heart are activated (Fig. 2). At the beginning of myocardial injury, the innate immune system is activated to allow the heart to adapt to increased stress in the short term [46]. However, this inflammatory response can become dysregulated, resulting in activation of the expression of certain proinflammatory cytokines. According to a previous study, TNF- $\alpha$ and IL-6 are expressed in the failing heart [47]. TNF- $\alpha$ can bind to TNF receptor 1 (TNFR1) in the cell membrane to activate the NADPH oxidase (NOX) complex. The activated NOX complex converts extracellular $\mathrm{O}_{2}$ into $\mathrm{O}_{2}{ }^{-}$and induces oxidative stress [48]. Finally, TNF-induced ROS production contributes to apoptosis by inducing mitochondrial outer-membrane permeabilization (MOMP) and Jun N-terminal kinase (JNK) activation [49]. Therefore, TNF- $\alpha$ can activate multiple signaling pathways to affect cardiomyocytes. Regarding other cytokines, the expression levels of both IL- $1 \alpha$ and IL- $1 \beta$ are upregulated in HF patients [50].

On the other hand, the adaptive immune system provides a highly specific response that is mediated by certain cellular components, such as activated $\mathrm{T}$ lymphocytes, macrophages and NK cells. These cells were observed to be increased in number in histological heart spec- imens from patients with chronic HF [51]. These activated macrophages, T cells and B cells can trigger a sustained inflammatory response by stimulating cytokine production, ultimately resulting in cytotoxicity and tissue injury [43].

\subsection{Cardiomyocyte death}

The loss of cardiomyocytes due to cell death, including apoptosis and necrosis, has been implicated in $\mathrm{HF}$ $[52,53]$, while inhibition of cardiomyocyte death has beneficial effects in the prevention of HF [54,55]; therefore, blocking cardiomyocyte death may be a new potential approach for the HF treatment. It was reported that FasL is expressed in HF patients [56]; furthermore, suppression of FasL has beneficial antiapoptotic effects and reduces the risk of death in HF patients $[57,58]$. On the other hand, the loss of mitochondrial cristae and destruction of the inner mitochondrial membrane were observed in vivo in animal models and in HF patients [59]. This process could mediate the cleavage of Bid and promote cytochrome c release. Finally, the induction of mitochondrial necrosis is triggered by the opening of the mitochondrial permeability transition pore (mPTP) due to $\mathrm{Ca}^{2+}$ influx. The loss of mitochondrial membrane potential and a decrease in ATP synthesis can result, eventually inducing necrotic cell death [60]. Moreover, inhibiting the opening of MPTP could attenuate myocardial necrosis induced by ischemia and oxidative stress [61].

\section{Short summary of the progress of understanding HF pathophysiology}

Considering the complex mechanisms of HF pathophysiology, it is clear that multiple signaling pathways and processes are involved in the induction of HF. More importantly, these processes do not function alone and are affected by each other. For instance, calcium abnormalities not only induce cardiomyocyte hypertrophy but also aggravate oxidative stress and increase ROS production. In addition, some proteins, such as Ang II and TNF- $\alpha$, have multiple effects on inflammation, fibrosis, and even cardiomyocyte death. In the early stage, oxidative stress is induced by the increased activity of certain enzymes and impaired antioxidant capacity. During this phase, the contractile dysfunction of cardiac myocytes is triggered by calcium abnormalities. Then, in the following stage, fibrosis and inflammation are induced, resulting in an increase in oxidative stress. Next, increased cardiomyocyte hypertrophy, apoptosis, and fibrotic changes in the extracellular matrix lead to the microstructural reorganization of cardiomyocytes. Although remarkable progress has been made in interventional and surgical methods in recent decades, the mortality rate of HF has not been reduced. Therefore, an increased understanding of the pathophysiological mechanisms behind the development of HF will help us to find novel, optimized pharmacotherapeutic targets. 


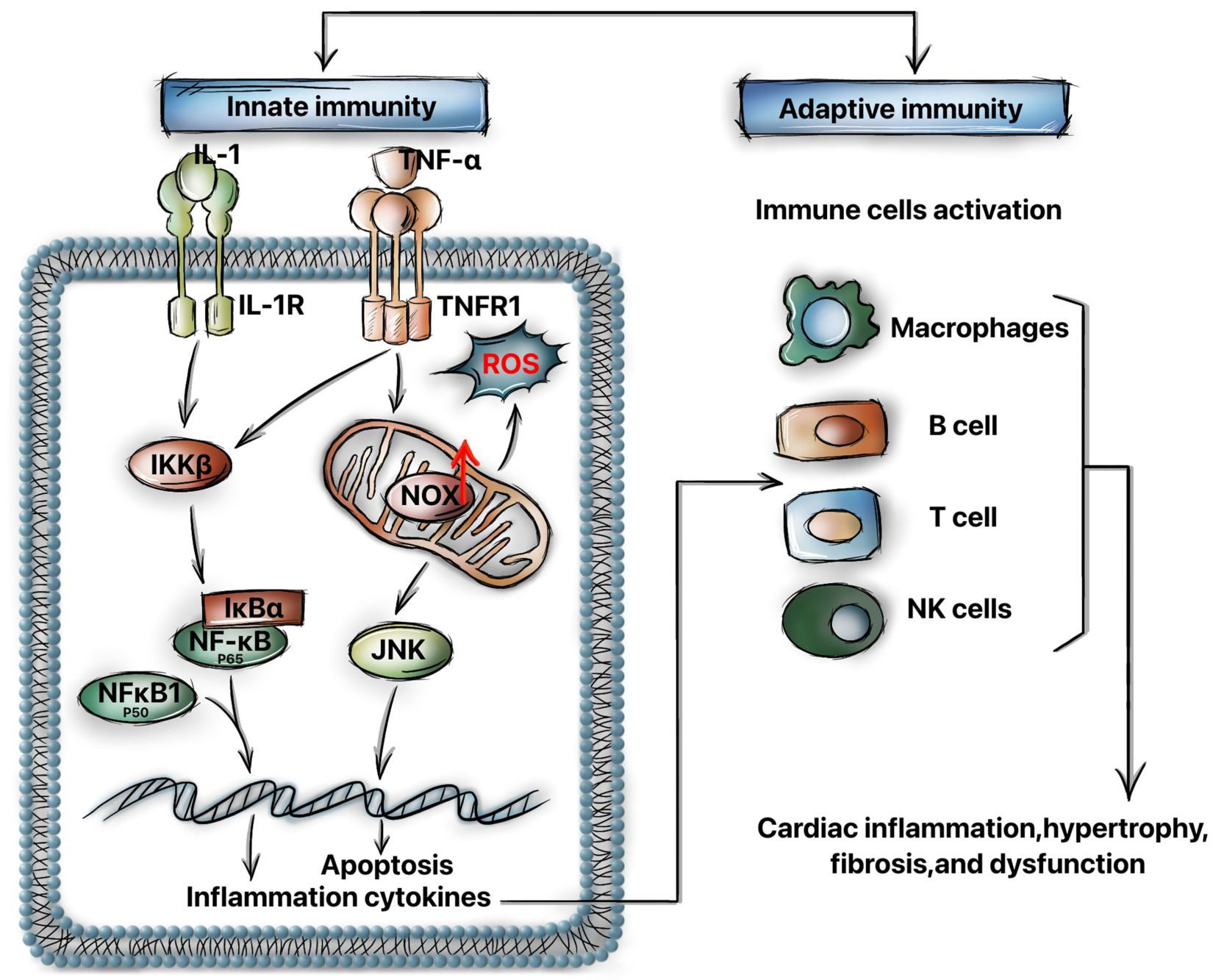

Fig. 2. The innate and adaptive immune systems are activated in heart failure. Acute injury, such as ischemia, pressure, or volume overload, can induce the binding of certain proinflammatory cytokines (TNF- $\alpha$ and IL-1) to their receptors, which activates the nuclear factor $-\kappa \mathrm{B}(\mathrm{NF}-\kappa \mathrm{B})$ signaling pathway to aggravate proinflammatory cytokine expression. These effects can also increase ROS production and lead to apoptosis. Finally, activation of the innate immune system triggers the activation of the adaptive immune response through the recruitment of immune cells, including macrophages, B cells, T cells and natural killer (NK) cells.

\section{Natural products from Chinese herbs to treat HF}

As mentioned above, Chinese herbs have been used for the effective management of HF for a long time. There are four different types of Chinese medicines that are being used in preclinical studies and in the clinic to treat HF, including TCM formulae, Chinese medicines, crude extracts of herbsand active components and compounds.

The herbs commonly used to treat HF are: Radix aconiti carmichaeli (Fuzi), Radix astragali (Huangqi), Panax ginseng (Renshen), Salvia miltiorrhiza (Danshen), etc. Chinese herbs have been successfully produced and made into the form of capsules, pills or injections, such as Qishenyiqi dripping pill (QSYQ), Danqi pill (DQP), Shengmai capsule, Shenmai injection, and Shengmai injection.
On the other hand, several herbs in different proportions can be prescribed together. These decoctions prescribed by physicians include: Baoyuan tang, Fuzhengkangfu Decoction, Shengmai san, Xuefuzhuyu tang and Zhenwu tang, etc. Compared to herbal compounds, active components and compounds can easily be investigated by scientifically rigorous clinical evaluations and some have been approved by the Food and Drug Administration (FDA) [62]. The different types of Chinese medicine used in the clinic and their components and sources are listed in Table 1.

\section{Clinical evaluations of natural products from Chinese herbs}

Although TCMs in China do not undergo the same rigorous testing procedures as Western drugs, a large number 
Table 1. Summary of the prescribed formulae and natural products from Chinese herbs used in clinical trials.

\begin{tabular}{|c|c|c|c|c|c|c|}
\hline $\begin{array}{l}\text { Medicine } \\
\text { form }\end{array}$ & Name & $\begin{array}{l}\text { Dosage } \\
\text { form }\end{array}$ & Prescription rights & Source & Major active compounds & Target disease \\
\hline \multirow[t]{7}{*}{$\begin{array}{l}\text { TCM } \\
\text { mula }\end{array}$} & Baoyuan tang & Decoction & TCM Physicians & $\begin{array}{l}\text { Panax ginseng, Radix astragali, Glycyrrhiza uralensis, Cin- } \\
\text { namomum cassia }\end{array}$ & Astragaloside IV, Ginsenoside, etc. & $\begin{array}{l}\text { Coronary disease, } \\
\text { Congestive heart failure }\end{array}$ \\
\hline & Danshen Yin & Decoction & TCM Physicians & Salvia miltiorrhiza, Santalum album, Amomum villosum & Tanshinone IIA, Salvianolic acid B, etc. & Coronary disease \\
\hline & $\begin{array}{l}\text { Fuzhengkangfu } \\
\text { Decoction }\end{array}$ & Decoction & TCM Physicians & $\begin{array}{l}\text { Radix codonopsis, Radix saposhnikoviae, Rhizoma atracty- } \\
\text { lodis macrocephalae, Poria, Radix morindae officinalis, Radix } \\
\text { glycyrrhizae }\end{array}$ & $\begin{array}{l}\text { Tangshenoside, Trametenolic acid, Arachic I } \\
\text { acid, etc. }\end{array}$ & $\begin{array}{l}\text { Non small cell lung } \\
\text { cancer, Heart failure }\end{array}$ \\
\hline & Shengmai San & Decoction & TCM Physicians & Panax ginseng, Ophiopogon japonicas, Schisandra chinensis & Ophiopogon saponin, Ginsenoside, etc. & $\begin{array}{l}\text { Coronary disease, } \\
\text { Congestive heart failure }\end{array}$ \\
\hline & $\begin{array}{l}\text { Taohongsiwu } \\
\text { Tang }\end{array}$ & Decoction & TCM Physicians & $\begin{array}{l}\text { Angelica sinensis, Ligusticum chuanxiong, Paeonia lactiflora, } \\
\text { Carthamus tinctorius, Radix rehmanniae preparata, Prunus } \\
\text { persica }\end{array}$ & $\begin{array}{l}\text { Polysaccharide, Iridoids, Ligustrazine, } \\
\text { Carthamin, Paeoniflorin, Amygdalin, etc. }\end{array}$ & $\begin{array}{l}\text { Coronary disease, } \\
\text { Cerebral infarction }\end{array}$ \\
\hline & $\begin{array}{l}\text { Xuefuzhuyu } \\
\text { Decoction }\end{array}$ & Decoction & TCM Physicians & $\begin{array}{l}\text { Prunus persica, Angelica sinensis, Carthamus tinctorius, } \\
\text { Radix paeoniae, Achyranthes bidentata, Ligusticum chuanx- } \\
\text { iong, Platycodon grandiflorus, Radix Bupleuri, Citrus auran- } \\
\text { tium, Rehmannia glutinosa, Glycyrrhiza uralensis }\end{array}$ & $\begin{array}{l}\text { Carthamin, Amygdalin, } \\
\text { Paeoniflorin, Triterpene saponins, Lgustrazine, } \\
\text { Saikoside, } \\
\text { Iridoids, Umbellifera lactone, etc. }\end{array}$ & $\begin{array}{l}\text { Coronary disease, } \\
\text { Congestive heart failure }\end{array}$ \\
\hline & Zhenwu Tang & Decoction & TCM Physicians & $\begin{array}{l}\text { Poria cocos, Paeonia lactiflora, Atractylodes macrocephala, } \\
\text { Zingiber officinale Roscoe, Radix Praeparata }\end{array}$ & $\begin{array}{l}\text { Ganodericacid, Paeoniflorin, Atractylol, Zin- } \\
\text { giberol, etc. }\end{array}$ & $\begin{array}{l}\text { Cardiogenic edema } \\
\text { heart failure }\end{array}$ \\
\hline \multirow[t]{3}{*}{$\begin{array}{l}\text { Chinese } \\
\text { medicine }\end{array}$} & Danqi Pill & $\begin{array}{l}\text { Guttate } \\
\text { Pills }\end{array}$ & $\begin{array}{l}\text { Western medicine/ } \\
\text { TCM Physicians }\end{array}$ & Salvia miltiorrhiza, Panax notoginseng, Borneol & Borneol, Notoginsenoside Tanshinone IIA, etc. & $\begin{array}{l}\text { Coronary disease, } \\
\text { Angina pectoris }\end{array}$ \\
\hline & $\begin{array}{l}\text { Danhong } \\
\text { Injection }\end{array}$ & Injection & $\begin{array}{l}\text { Western medicine/ } \\
\text { TCM Physicians }\end{array}$ & Salvia miltiorrhiza, Carthamus tinctorius & Carthamin, Tanshinone IIA, etc. & $\begin{array}{l}\text { Coronary disease, } \\
\text { Angina pectoris, } \\
\text { Myocardial infarction }\end{array}$ \\
\hline & $\begin{array}{l}\text { Qili Qiangxin } \\
\text { Capsule }\end{array}$ & Capsule & $\begin{array}{l}\text { Western medicine/ } \\
\text { TCM Physicians }\end{array}$ & $\begin{array}{l}\text { Radix astragali, Ginseng, Aconitum carmichaelii Debx, Salvia } \\
\text { miltiorrhiza, Lepidium virginicum, Alisma orientalis, Polygo- } \\
\text { natum odoratum, Cinnamomum cassia Presl, Carthamus tinc- } \\
\text { torius, Periploca sepium, Citrus reticulata Blanco }\end{array}$ & $\begin{array}{l}\text { Astragaloside IV, Ginsenoside, Aconitine, Tan- } \\
\text { shinone IIA, Alisol, Carthamin, Cinnamalde- } \\
\text { hyde, Hesperidin, etc. }\end{array}$ & $\begin{array}{l}\text { Coronary disease, } \\
\text { Congestive heart failure }\end{array}$ \\
\hline
\end{tabular}


Table 1. Continued.

\begin{tabular}{|c|c|c|c|c|c|c|}
\hline Medicine form & Name & Dosage form & Prescription rights & Source & Major active compounds & Target disease \\
\hline & Qishen Yiqi & Guttate Pills & Western medicine/ & Radix astragali, Salvia miltiorrhiza, & Astragaloside IV, Tanshinone IIA, & Coronary disease, Angina pectoris \\
\hline & Dripping Pill (QSYQ) & & TCM Physicians & Panax notoginseng, Dalbergia odorifera & Duartin, Ginsenoside, etc. & \\
\hline & Shenmai Injection & Injection & TCM Physicians & Panax ginseng, Ophiopogon japonicus & Ginsenoside, Ophiopogonin, etc. & Coronary disease, Viral myocarditis \\
\hline & Shengmai Injection & Injection & TCM Physicians & $\begin{array}{l}\text { Panax ginseng, Ophiopogon japonicas, } \\
\text { Schisandra chinensis }\end{array}$ & $\begin{array}{l}\text { Ginsenoside, Ophiopogonin, } \\
\text { Schizandrin, etc. }\end{array}$ & $\begin{array}{l}\text { Myocardial infarction, } \\
\text { Chronic heart failure }\end{array}$ \\
\hline \multirow{3}{*}{$\begin{array}{l}\text { Crude extract of } \\
\text { herb }\end{array}$} & Di'ao Xinxuekang & Capsule & Western medicine/ & Dioscorea panthaica & Steroidal saponins & Coronary disease, \\
\hline & Capsule & & TCM Physicians & & & Chronic heart failure, \\
\hline & Huangqi Injection & Injection & TCM Physicians & Radix astragali & Astragaloside IV (AS-IV) & Viral myocarditis \\
\hline \multirow{11}{*}{$\begin{array}{l}\text { Active component } \\
\text { and compound }\end{array}$} & Adoniside & Tablet & Western medicine/ & Adonis vernalis & Adoniside & Acute and chronic heart failure \\
\hline & & & TCM Physicians & & & \\
\hline & Astragaloside IV (AS-IV) & Tablet & Western medicine/ & Radix astragali & Astragaloside IV(AS-IV) & Chronic heart failure \\
\hline & & & TCM Physicians & & & \\
\hline & Digitoxin & Tablet/Injection & Western medicine/ & Digitalis lanata & Digitoxin & Coronary disease \\
\hline & & & TCM Physicians & & & Chronic heart failure \\
\hline & Tanshinone IIA & Injection & TCM Physicians & Salvia miltiorrhiza & Tanshinone IIA & $\begin{array}{l}\text { Acute myocardial infarction, severe } \\
\text { heart failure }\end{array}$ \\
\hline & Ibopamine & Tablet & Western medicine/ & Plectranthus barbatus & Ibopamine & Heart failure \\
\hline & & & TCM Physicians & & & \\
\hline & Ouabain & Tablet & Western medicine/ & Strophanthus gratus & Ouabain & Coronary disease \\
\hline & & & TCM Physicians & & & Congestive heart failure \\
\hline
\end{tabular}


of randomized controlled trials were carried out to assess the efficacy of TCMs to treat HF. Some of these TCMs have already been put into clinical evaluations.

Di'ao Xinxuekang capsule is extracted from the rhizomes of Dioscorea panthaica. A randomized control trial showed that the proportion of angina pectoris patients was significantly reduced after 20 weeks of treatment with Di'ao Xinxuekang capsule [63]. Furthermore, a randomized clinical trial demonstrated that Di'ao Xinxuekang capsule seemed to have a better protective effect on HF patients than isosorbide dinitrate [64].

QSYQ, a patented Chinese prescription, has been applied for the integrative treatment of HF patients. A randomized clinical trial demonstrated that 12 months of QSYQ treatment had effects similar to those of aspirin for the treatment of myocardial infarction [65].

Qili qiangxin capsule was approved for the treatment of $\mathrm{HF}$ in 2004. It contains extract of 11 Chinese herbs, as shown in Table 1. In a clinical trial, N-terminal prohormone of brain natriuretic peptide (NT-proBNP) levels were downregulated by Qili qiangxin capsule and its performance was superior compared with the placebo [66].

Huangqi injection is an extract of Radix astragali that is also commonly used to treat chronic HF in the clinic. It was found that Huangqi injection has a beneficial effect on improving heart function, such as increased left ventricular ejection fraction (LVEF) and decreased stroke volume (SV) levels [67]. On the other hand, it was found that the expression levels of sIL-2R, IgG and IgA were reduced in congestive HF patients after Huangqi injection treatment which suggested that immune function was also rescued [68].

Shenmai injection is extracted from Panax ginseng. A double-blind, multicenter study showed that the Shenmai injection group demonstrated a significant improvement in heart function compared with the placebo group [69]. On the other hand, Shenmai injection also improved left ventricular diastolic function (LVDF) in chronic HF patients [70]. Finally, Shenmai injectionreduced the expression levels of plasma neuropeptide Y (NPY), endothelin (ET) and atrial natriuretic polypeptide (ANP) to improve the cardiac function in HF patient [71].

Shengmai injection is extracted from Panax ginseng, Ophiopogon japonicas and Schisandra chinensis. It was reported that Shengmai injection could improve heart function in HF patients and inhibit myocardial cell apoptosis by reducing the death receptor of soluble tumor necrosis factor related apoptosis inducing ligand (TRAIL) [72]. The levels of plasma P-selectin, von Willebrand factor (vWF) and Ddimer in HF patients were also reduced after Shengmai injection which suggests that cardiac function was improved [73].

Digitoxin, a traditional Chinese medicine monomer extracted from Digitalis lanata Ehrh (Maohua Yangdihua) has been commonly applied in the treatment of HF patients. Several double-blind placebo-controlled trials have proven the efficacy of digoxin in the treatment of HF [74-76]. From the PROVED trial, a significant worsening of exercise duration occurred in patients on placebo medication when compared with the digoxin group. Moreover, the left ventricular ejection fraction was increased in the digoxin group and decreased in the placebo group [74]. A randomized, placebo-controlled clinical trial enrolled 3505 patients and revealed that digitoxin could reduce the risks of mortality and hospital admission for HF patients [75]. On the other hand, it was shown that the differences in chronic HF scores during the digoxin and digitoxin periods were not significant [76]. In addition to digitoxin, other natural products extracted from Digitalis lanata, including lanatoside A/C, were used to treat HF in clinical trials [77,78].

Tanshinone IIA is a potent pharmacological compound extracted from Salvia miltiorrhiza (Danshen). Tanshinone IIA has a similar therapeutic effect on myocardial infarction as alprostadil; however, the cardiac functions of all patients who were treated with Tanshinone IIA injection were significantly improved [79]. As a derivative of Tanshinone IIA, sodium Tanshinone IIA sulfonate (STS) also has a cardioprotective effect. In a randomized clinical trial, 101 myocardial infarction patients were immediately assigned to receive STS or saline control, and 6months of treatment with STS inhibited the damage to the infarcted myocardium by significantly reducing the level of neutrophils [80].

Astragaloside IV(AS-IV) is the main component of Radix Astragali (Huangqi) which is widely used among patients with chronic HF in the clinic [81]. Alleviation was shown in 15 congestive heart failure patients, and their exercise capability was reinforced after AS-IV injection [82]; however, there have been no direct clinical trials to explore the cardioprotective effect of adoniside.

Ibopamine is extracted from Plectranthus barbatus. A double-blind placebo-controlled study showed that ibopamine inhibited neurohumoral activation in patients with mild to moderate chronic HF [83].

\section{The mechanisms by which natural products from Chinese herbs treat $\mathrm{HF}$}

The pharmacological mechanisms by which these natural products treat HF have been explored in numerous studies [84]. Experimental studies have demonstrated that natural products from Chinese herbs have anticardiac hypertrophy, antioxidant, antifibrotic, antiinflammatory and antiapoptotic properties (Table 2, Ref. [85-135]). As discussed above, these biological pathways are interconnected, and these natural products may function in a multicomponent and multitarget manner.

\subsection{Anticardiac hypertrophy effects}

Although it was reported that Zhenwu Tang could reduce the weight and volume of the heart and improve cardiac function, the mechanism is not clear [85]. Another 
Table 2. The mechanisms by which the natural products from Chinese herbs treat HF.

\begin{tabular}{|c|c|c|c|c|}
\hline Property & Natural products & Targets or pathways & Type of study & References \\
\hline \multirow[t]{10}{*}{ Inhibiting hypertrophy } & Zhenwu Tang & p-ERK1/2 $\downarrow$, p-p38 $\downarrow$, p-JNK $\downarrow$ and BNP $\downarrow$ & Constricting the abdominal aorta in Rat & {$[85,86]$} \\
\hline & Danhong injection & p38 and NF- $\kappa$ b pathway $\downarrow \rightarrow$ ANP and BNP $\downarrow$ & ISO in $\mathrm{H} 9 \mathrm{C} 2$ and rat & {$[87]$} \\
\hline & Shengmai injection & AMPK signaling pathway $\uparrow$ & Angiotensin II in primary cardiomyocyte & [88] \\
\hline & Digitoxin & $\mathrm{Na}_{+} / \mathrm{Ca}_{2+}$ exchanger levels $\uparrow$, SERCA $2 \uparrow$ & Coronary artery ligation in rats & [89] \\
\hline & Tanshinone IIA & Voltage-gated $\mathrm{Ca}_{2}+$ channels $\downarrow$ & Systolic blood pressure in rat & [90] \\
\hline & & Store-operated $\mathrm{Ca}_{2+}$ channels (SOCC) & Hypoxia in smooth muscle cells & [91] \\
\hline & & MAPKs and IGF-2R pathway $\downarrow$ & Angiotensin II in $\mathrm{H} 9 \mathrm{C} 2$ cells & [92] \\
\hline & Astragaloside IV(AS-IV) & $\mathrm{CaSR} / \mathrm{PLC} / \mathrm{PKC} \alpha / \mathrm{PP} 1 \downarrow \rightarrow \mathrm{SERCA} 2 \alpha \uparrow$ & Ligaturing LAD in rats & [93] \\
\hline & & Nrf2 $\uparrow \rightarrow$ TBK1/PI3K/AKT activity $\downarrow$ & Aortic banding in mice & {$[94,95]$} \\
\hline & & TLR4/NF-кB $\downarrow$ & ISO in rat & {$[96]$} \\
\hline \multirow[t]{11}{*}{ Anti-oxidation } & Shengmai San & MDA $\downarrow$ & Ligaturing $\mathrm{LAD}$ in rat & [97] \\
\hline & Danqi Pill & HIF- $1 \alpha /$ PGC- $1 \alpha$ signaling pathway $\uparrow$ & Ligaturing $\mathrm{LAD}$ in rat, $\mathrm{OGD} / \mathrm{R}$-induced $\mathrm{H} 9 \mathrm{C} 2$ cells & [98] \\
\hline & Shengmai injection & $\mathrm{AKT}$ and $\mathrm{ERK} 1 / 2 \rightarrow \mathrm{SOD} \uparrow$ & $\mathrm{H} 2 \mathrm{O} 2$ in $\mathrm{n}$ primary cardiomyocyte & [99] \\
\hline & Tanshinone IIA & $\mathrm{GPx} \uparrow \rightarrow \mathrm{SOD} \uparrow, \mathrm{MDA} \downarrow$ & $\mathrm{H} 2 \mathrm{O} 2$ in macrophages & {$[100]$} \\
\hline & & $\operatorname{Nrf} 2 \uparrow$ & $\mathrm{H} 2 \mathrm{O} 2$ in $\mathrm{H} 9 \mathrm{C} 2$ cells & [101] \\
\hline & & NADPH oxidase $\uparrow$ & Ligaturing LAD in rats & {$[102]$} \\
\hline & Astragaloside IV(AS-IV) & $\mathrm{NOXs} \downarrow \rightarrow \mathrm{SOD} \uparrow$ & ApoE-/- mice, ISO in primary rat & {$[103,104]$} \\
\hline & & $\mathrm{MDA}, \mathrm{CPK}, \mathrm{LDH} \downarrow$ & $\mathrm{H} / \mathrm{R}$ in cardiomyocyte & [105] \\
\hline & & NADPH oxidase-ROS-NF- $\kappa \mathrm{B} \downarrow$ & $\mathrm{H} 2 \mathrm{O} 2$ in HUVECs & [106] \\
\hline & Ouabain & $\mathrm{PI} 3 \mathrm{~K} / \mathrm{Akt} \uparrow \rightarrow \mathrm{NO} \uparrow$ & Ligaturing LAD in rats & {$[107]$} \\
\hline & & NADPH oxidase $\uparrow$ & Primary cardiomyocytes & [108] \\
\hline \multirow[t]{11}{*}{ Anti- fibrosis } & Danshen formulae & NOX2/ROS/p38 $\rightarrow$ Collagen I $\downarrow$ & ISO in rat & [109] \\
\hline & Shengmai San & Collagen I, Collagen III $\downarrow$ & Myocardial infarction (MI) in rat & [110] \\
\hline & Xuefuzhuyu Decoction & TGF- $\beta 1 \downarrow$ & Spontaneously hypertensive rats & [111] \\
\hline & Qishen Yiqi Dripping Pill (QSYQ) & $\begin{array}{l}\text { RAAS } \downarrow \rightarrow \text { MMP-2,MMP-9, } \\
\text { Collagen I, Collagen III } \downarrow\end{array}$ & Ligaturing LAD in rats & [112] \\
\hline & Shengmai & Collagen I, Collagen III $\downarrow$, TGF- $\beta 1 \downarrow$ & Doxorubicin injection in rat & [113] \\
\hline & Tanshinone IIA & $\begin{array}{l}\text { NADPH oxidase } \uparrow \rightarrow \text { collagen I, } \\
\text { collagen III, TGF- } \beta 1 \downarrow\end{array}$ & Ligaturing LAD in rats & [102] \\
\hline & & NO $\uparrow$, eNOS phosphorylation $\uparrow$ & Angiotensin II in rat cardiac fibroblasts & [114] \\
\hline & & $\mathrm{NOX} \downarrow$ & LPS in mice & [115] \\
\hline & Astragaloside IV(AS-IV) & TRPM7 $\downarrow \rightarrow$ Collagen $\downarrow$ & ISO in rats; Hypoxia or ISO in fibroblasts & [116] \\
\hline & & ROS-mediated MAPK $\downarrow$ & ISO in rat & [105] \\
\hline & & ROS $\downarrow \rightarrow$ cardiotrophin- $1 \downarrow \rightarrow$ Collagen $\downarrow$ & ISO in cardiac fibroblasts & {$[117]$} \\
\hline
\end{tabular}


Table 2. Continued.

\begin{tabular}{|c|c|c|c|c|}
\hline Property & Natural products & Targets or pathways & Type of study & References \\
\hline \multirow[t]{10}{*}{ Anti-inflammtion } & \multirow[t]{3}{*}{ Danqi pill } & PLA2, COX-2, NF-кB $\downarrow$ & Ligaturing LAD in rat & [118] \\
\hline & & HIF- $1 \alpha /$ PGC- $1 \alpha$ signaling pathway $\uparrow$ & Ligaturing LAD in rat & [98] \\
\hline & & TXB2 $\downarrow$, PGI $2 \uparrow$ & Ligaturing LAD in rat & {$[119]$} \\
\hline & \multicolumn{2}{|c|}{ Qishen Yiqi Dripping Pill (QSYQ) NF- $\kappa \mathrm{B} \downarrow, \mathrm{JAK} 1 \downarrow$ and AKT $\downarrow$} & Ligaturing LAD in rats & [112] \\
\hline & Danhong injection & NF- $\kappa \mathrm{B} \downarrow \rightarrow \mathrm{TNF}-\alpha, \mathrm{IL}-1 \beta$, and IL- $6 \downarrow$ & Ligaturing LAD in rat & [120] \\
\hline & \multirow[t]{2}{*}{ Tanshinone IIA } & TLR4-NF- $\mathrm{B} \downarrow$ & Ligaturing LAD in mice & [121] \\
\hline & & SIRT $1 \uparrow \rightarrow$ TNF- $\alpha$ and IL- $6 \downarrow$ & $\mathrm{TAC}$ in rat & [122] \\
\hline & \multirow[t]{2}{*}{ Astragaloside IV(AS-IV) } & TLR4/NF-кB $\downarrow$ & ISO in rats & {$[96,123]$} \\
\hline & & NF-кB signaling $\downarrow$, PI3K/AKT $\uparrow \rightarrow \mathrm{TNF}-\alpha \downarrow$ & LPS in mice & [124] \\
\hline & Ouabain & IL-6 $\downarrow$, TNF-alpha $\downarrow$ & LPS in mice & [125] \\
\hline \multirow{9}{*}{ Anti-cardiomyocyte death } & Fuzhengkangfu decoction & Bcl- $2 \uparrow$, cleaved caspase- $3 \downarrow$ & Doxorubicin in $\mathrm{H} 9 \mathrm{C} 2$ & [126] \\
\hline & Qili Qiangxin Capsule & Fas $\downarrow$, cleaved caspase- $3 \downarrow$ & Ligaturing LAD in rat & [127] \\
\hline & Shengmai injection & ER stress $\downarrow \rightarrow$ caspase- $12 \downarrow$ & Doxorubicin in rat & {$[128]$} \\
\hline & \multirow[t]{3}{*}{ Tanshinone IIA/ } & Bcl-X $\downarrow$, cleaved caspase- $3 \downarrow$ & Abdominal aortic coarctation in rat, Doxorubicin in cardiomyocyte & {$[129,130]$} \\
\hline & & Nrf2 $\uparrow \rightarrow \mathrm{p} 38$ and mTOR signaling $\downarrow$ & $\mathrm{TAC}$ in mice & [131] \\
\hline & & AMPA $\uparrow \rightarrow$ Bax, cleaved caspase- $3 \downarrow$ & LAD in rat, $\mathrm{H} 2 \mathrm{O} 2$ in $\mathrm{H} 9 \mathrm{C} 2$ & [132] \\
\hline & \multirow[t]{3}{*}{ Astragaloside IV(AS-IV) } & Calpain-1 and ROS $\downarrow \rightarrow$ Bcl-2/Bax $\uparrow$, MMP $\uparrow$ & ISO in rats or $\mathrm{H} 9 \mathrm{C} 2$ cells & [133] \\
\hline & & $\mathrm{PI} 3 \mathrm{~K} / \mathrm{AKT} \uparrow$ & Doxorubicin in cardiomyocyte & {$[134]$} \\
\hline & & Senp $1 \downarrow \rightarrow$ Bcl-2/bax $\uparrow$, cleaved caspase-3 $\downarrow$ & LAD in mice & [135] \\
\hline
\end{tabular}

AKT, protein kinase B; AMPK, AMP-activated protein kinase; ANP, atrial natriuretic peptide; BNP, B-type natriuretic peptide; CaSR, calcium-sensing receptor; COX-2, cyclooxygenase 2; ERK1/2, extracellular signal-regulated kinase 1/2; GPx, glutathione peroxidase; HIF-1 $\alpha$, hypoxia-inducible factor-1alpha; IGF-2R, insulin-like growth factor 2 receptor; ISO, isoproterenol; JAK1, Janus kinase-1; JNK, Jun N-terminal kinase; LAD, the coronary artery; MAPKs, mitogen-activated protein kinases; MDA, malondialdehyde; MMP, matrix metalloproteinase; mTOR, mammalian target of rapamycin; NF-кB, nuclear factor kappa B; NO, nitric oxide; NOX, NADPH oxidase; Nrf2, nuclear factor erythroid 2-related factor; PGC-1 $\alpha$, peroxisome proliferator-activated receptorgamma coactivator-1alpha; PGI2, prostaglandin I2; PI3K, Phosphatidylinositol-4,5-bisphosphate 3-kinase; PKC- $\alpha$, protein kinase C- $\alpha$; PLA2, phospholipase A2; PLC, phospholipase C; PP-1, Protein phosphatase-1; ROS, reactive oxygen species; RAAS, renin-angiotensin-aldosterone system; SERCA2a, sarcoendoplasmic reticulum calcium ATPase; Senp1, small ubiquitin-like modifier (SUMO)-specific protease 1; SIRT1, silent information regulator 1; SOD, superoxide dismutase; TBK1, TANK-binding kinase 1; TGF $\beta$-1, transforming growth factor $\beta$-1; TLR4, toll-like receptor 4; TNF- $\alpha$, tumour necrosis factor; TRPM7, transient receptor potential melastatin 7; TXB2, thromboxane B2. Up arrows indicate increased activity or production. Down arrows indicate decreased activity or production. 
study showed that Zhenwu Tang delayed ventricular hypertrophy by suppressing the expression of BNP, pextracellular signal-regulated kinase $1 / 2$ (ERK1/2), p-p38 and p-JNK [86].

According to previous study, the mass of the left ventricle (LV) and the thickness of the LV end-systolic posterior wall (LVPWs) in isoproterenol (ISO)-induced rats were reduced after Danghong injection which was associated with a reduction in atrial natriuretic peptide (ANP) and brain natriuretic peptide (BNP) [87]. On the other hand, Danghong injection significantly attenuated cardiac hypertrophy by suppressing cell area enlargement and downregulating ET-1-induced brain natriuretic peptide (BNP) and cardiac muscle troponin $\mathrm{T}$ (TNNT2) protein expression [136]. In addition, it was reported that Shengmai injection could improve the cell-survival rate and reduce myocardial cell hypertrophy via activation of the AMP-activated protein kinase (AMPK) signaling pathway [88].

The intracellular calcium level is an important hypertrophic messenger and there are many $\mathrm{Ca}^{2+}$ channels that regulate intracellular calcium levels. In rats with $\mathrm{HF}$, digitoxin mitigated myocardial remodeling and contractile impairment via $\mathrm{Na}^{+} / \mathrm{Ca}^{2+}$ exchanger levels, and decreased SERCA [89].

Tanshinone IIA has the ability to open ATP-sensitive $\mathrm{K}^{+}$channels which could result in decreased $\mathrm{Ca}^{2+}$ levels in the cytoplasm and ultimately lead to vasodilatation [90]. On the other hand, it has been shown that sodium tanshinone IIA sulfonate (STS), a derivative of Tanshinone IIA, can inhibit $\mathrm{Ca}^{2+}$ entry through reducing store-operated $\mathrm{Ca}^{2+}$ channels (SOCCs) and the expression of transient receptor potential canonical proteins (TRPCs) [91]. Tan IIA can also alleviate Ang II-induced hypertrophy in myocytes; however, multiple signaling pathways are involved, such as the MAPK and insulin-like growth factor 2 receptor (IGF2R) pathways [92].

AS-IV improved cardiac diastole in chronic HF by decreasing the expression of protein kinase C- $\alpha$ (PKC- $\alpha)$, reducing PP-1 activity and enhancing SERCA activity [93]. On the other hand, it was reported that As-IV significantly improved cardiac function and limited hypertrophy by suppressing TANK-binding kinase 1 (TBK1)/ phosphatidylinositol - 3-kinase (PI3K)/protein kinase B (AKT) activity [94] which was partially mediated by activating the nuclear factor erythroid 2-related factor (Nrf2)/HO-1 signaling pathway [95]. Finally, AS-IV suppressed hypertrophyrelated parameters and Toll-like receptor 4 (TLR4)/nuclear factor kappa B (NF- $\kappa \mathrm{B})$ in a rat model of ISO-induced cardiac hypertrophy [96]. This result indicated that AS-IV could inhibit myocardial hypertrophy by suppressing inflammation.

\subsection{Anti-oxidative properties}

As discussed above, oxidative stress plays a key role in the development of HF. It was reported that
YiQiFuMai powder injection (YQFM), a traditional Chinese medicine prescription redeveloped based on ShengMai-San, could decrease the content of malondialdehyde (MDA) [97]. Moreover, Danqi Pill protects against HF induced myocardial infarction by improving myocardial glucose metabolism, mitochondrial oxidative phosphorylation and biogenesis, which is associated with regulation of the hypoxia-inducible factor-1alpha (HIF-1 $\alpha$ )/peroxisome proliferator-activated receptor-gamma coactivator-1alpha (PGC-1 $\alpha$ ) signaling pathway [98]. Furthermore, Shengmai injection restored the activity of superoxide dismutase (SOD), and glutathione reductase (GSR) in cardiomyocytes with $\mathrm{H}_{2} \mathrm{O}_{2}$-induced oxidative stress by activating AKT and the ERK1/2 pathway [99].

According to Chen et al. [137], Tanshinone IIA reduces the level of MDA and elevates the level of SOD, which is consistent with our previous study [138]. Furthermore, Tanshinone IIA can prevent oxidative stress injury by increasing glutathione peroxidase (GPx) activity and mRNA levels [100]. Although there is no direct evidence for the underlying mechanism, some studies have reported that Tan IIA can induce the expression of nuclear factor (erythroid-derived 2)-like 2 (Nrf2), a redox-sensitive transcription factor, to attenuate oxidative stress injury via increased transcription of certain antioxidant genes, including SOD [101]. Finally, a recent study showed that Tanshinone IIA reversed the decreases in superoxide dismutase activity and malondialdehyde and the increases of superoxide anions and NADPH oxidase activity in both HF rats and Ang IItreated cardiac fibroblasts [102].

It was reported that AS-IV treatment could attenuate oxidative stress via increasing the activity of SOD and reducing nicotinamide adenine dinucleotide phosphate oxidase (NOX) levels [103]. On the other hand, AS-IV could markedly enhanced cell viability and reduced MDA content and lactate dehydrogenase (LDH) activity in cardiomyocytes a hypoxia/reoxygenation (H/R) model [104]. ASIV could also block ROS production in ISO-treated primary rat cardiac fibroblasts which might be associated with inhibiting nicotinamide adenine dinucleotide phosphate (NADPH) oxidase-NF- $\kappa$ B signaling $[105,106]$.

Ouabain is an endogenous cardiac glycoside produced in the central nervous system that can also be extracted from the seeds of Strophanthus gratus [139]. It was reported that ouabain could decrease the vascular reactivity of aortic rings and increase nitric oxide production via a PI3K/Aktdependent pathway in HF rats [107]. On the other hand, ouabain could also stimulate mitochondrial NADH production to attenuate the oxidative metabolic state [108].

\subsection{Anti-fibrotic effects}

Several TCM formulae have been shown to have anti-fibrotic effects. Isopropyl 3-(3,4-dihydroxyphenyl)-2hydroxylpropanoate (IDHP), a metabolite of Danshen formulae, attenuates cardiac fibrosis and decreases collagen I 
synthesis via a NOX2/ROS/p38 pathway [109]. Additionally, a recent study showed that a Shengmai San-derived herbal improved the conduction velocity and homogeneity and decreased left atrial positive fibrosis areas and the expression of type I and III collagen in myocardial infarction (MI)-induced HF rats [110]. Finally, a study showed that Xuefuzhuyu decoction treatment decreased cardiac fibrosis induced by hypertension and inhibited the expression of TGF- $\beta 1$ [111]

QSYQ exerted an anti-fibrotic effect in AMI rat models by reducing matrix metalloproteinase-2 (MMP-2), MMP-9, collagen I and collagen III by inhibiting the reninangiotensin-aldosterone system (RAAS) [112]. Other studies confirmed that QSYQ had definite effect on myocardial fibrosis via the TGF $\beta 1$ signaling pathway [140]. Furthermore, Shengmai has protective effect on reducing cardiac toxicity in rats treated with doxorubicin (DOX). Myocardial fibrosis markers, including procollagen type III and TGF$\beta 1$, were suppressed after Shengmai treatment [113].

According to a previous study, Tan IIA administration markedly reversed the increases of expression levels of collagen I, III, TGF $\beta 1, \alpha$ SMA, MMP- 2 and MMP-9 in a rat model of HF [102]. Although the underlying mechanism is not clear, it is associated with the activation of Smad2/3, $\mathrm{NF}-\kappa \mathrm{B}$ and Nrf2 expression, which not only has an antioxidant effect but also attenuates fibrosis [141]. Furthermore, Tan IIA inhibits Ang II-induced cell proliferation in rat cardiac fibroblasts by increasing NO generation and eNOS phosphorylation [114]. Finally, Tan IIA attenuates the development of myocardial fibrosis and fibrosis-related genes and mediators by decreasing the expression of NADPH oxidase subunit (NOX) and ROS levels [115]. However, the anti-fibrotic effect mainly involves other organs, such as the lungs and kidney; therefore, the anti-fibrotic effects of Tan IIA on cardiac tissue need to be further investigated.

According to a previous study, AS-IV inhibited transient receptor potential melastatin 7 (TRPM7) protein expression and blocked hypoxia-induced fibrosis to attenuate fibrosis of the heart tissue [116]. On the other hand, ASIV significantly inhibited ISO-induced fibrosis by blocking MAPK activation in rat cardiac fibroblasts [105]. Finally, cardiac fibroblast proliferation and type I collagen synthesis were effectively abrogated by cardiotrophin-1 (CT-1) small interfering RNA. This result suggested that AS-IV could also prevent cardiac fibrosis by inhibiting CT-1 upregulation [117].

\subsection{Anti-inflammatory effects}

According to a previous study, the Danqi pill downregulated the expression of phospholipase A2 (PLA2), cyclooxygenase $2(\mathrm{COX}-2)$ and NF- $\kappa \mathrm{B}$ in the inflammatory pathway in a model of coronary heart disease [118]; however, the underlying mechanism remains elusive. A recent study showed that the Danqi pill could improve cardiac functions and attenuate inflammatory infiltration by regu- lating HIF-1 $\alpha /$ PGC-1 $\alpha$ signaling pathway in HF post-AMI rats [98]. Furthermore, the Danqi pill downregulated the expression of thromboxane B2 (TXB2) and upregulated the expression of prostaglandin I2 (PGI2) to suppress inflammation [119].

Furthermore, QSYQ attenuated myocardial fibrosis by reducing the expression of the angiotensin type 1 receptor (AT1), increasing the expression of AT2 in the RAAS pathway and suppressing the levels of NF- $\kappa \mathrm{B}, \mathrm{JAK} 1$ and AKT [112]. Finally, Danhong injection also suppressed the expression of inflammatory factors and inhibited NF$\kappa \mathrm{B}$ p65 nuclear translocation and phosphorylation of $\mathrm{I} \kappa \mathrm{B} \alpha$ to exert anti-inflammatory effects, which were associated with the regulation of miRNA expression [120]. In a clinical trial, inflammatory factor-soluble CD40 ligand was also reduced by Danhong injection in acute coronary syndrome (ACS) patients [142].

Tanshinone IIA has been used in TCM for the treatment of a variety of inflammatory and cardiovascular disorders. According to a previous study, Tan-IIA can attenuate LADinduced myocardial ischemia injury by inhibiting the expression of certain proinflammatory cytokines (IL$1 \beta$ and TNF- $\alpha$ ) associated with inhibiting Toll-like receptor (TLR) 4-NF- $\kappa$ B signaling [121]. Furthermore, Tan IIA significantly alleviates transverse aortic constriction-induced myocardial remodeling by activating the silent information regulator 1 (SIRT1) signaling pathway and then reduces myocardium malondialdehyde (MDA) content and cardiac inflammatory cytokines (TNF- $\alpha$ and IL-6) [122]. In addition to suppressing the expression of proinflammatory cytokines, Tanshinone IIA can also elevate the levels of antiinflammatory cytokines, such as IL-10, to suppress T cell activation [143].

AS-IV has a strong effect against inflammation. ASIV treatment inhibited the highly activated inflammatory response by suppressing the TLR4/NF- $\kappa$ B pathway in HF rats $[96,123]$. In LPS-induced HF mice, AS-IV significantly decreased serum inflammatory mediators, including TNF$\alpha$ and monocyte chemoattractant protein-1 (MCP-1), by suppressing NF- $\kappa$ B signaling and activating the PI3K/AKT pathway [124].

Ouabain was found to protect against LPS-induced inflammation in mice and decrease the expression levels of IL-6 and TNF-alpha in vivo [125]; however, a recent study demonstrated that ouabain could induce NLRP3 inflammasome activation as well as subsequent IL- $1 \beta$ release from macrophages and then promote cardiac inflammation [144]. Thus, the underlying adverse effects of cardiac glycosides needed to be further investigated.

\subsection{Anti-cardiomyocyte death effects}

According to our study, Fuzhengkangfu decoction ameliorated DOX-induced $\mathrm{H} 9 \mathrm{C} 2$ cell apoptosis and increased the Bcl-2/Bax ratio which might be associated with inhibiting the loss of mitochondrial membrane potential 
[126]. Qili qiangxin capsule inhibited cardiomyocyte apoptosis in ischemic heart tissues by suppressing the expression of Fas and caspase-3 [127]. QishenYiqi Dripping Pill also efficiently improved cardiac function by inhibiting cardiac apoptosis via the $\beta 2$-adrenergic receptor signaling pathway [145]. Shengmai injection inhibited endoplasmic reticulum stress-induced apoptosis and rescued heart dysfunction induced by DOX [128]. On the other hand, one study showed that Shengmai injection reduced apoptotic cardiomyocytes induced by $\mathrm{H}_{2} \mathrm{O}_{2}$ by upregulating intracellular AKT phosphorylation and downregulating ERK1/2 phosphorylation [99].

Several studies have reported that Tan IIA plays a critical role in attenuating cardiomyocyte apoptosis in models of HF $[129,130]$. Specifically, Tan IIA can protect cardiomyocytes from doxorubicin-induced apoptosis by reducing the levels of cleaved caspase- 3 and cytosolic cytochrome c, which are associated with the activation of AKT signaling pathways and suppression of ROS generation [130]. Indeed, our previous study showed that Tan IIA can increase the activity of SOD [146]. Although the underlying mechanism is not clear, it might be associated with an increase in Nrf2 gene transcription. It has been demonstrated that Tan IIA can increase Nrf2 expression to reduce myocardial apoptosis and that this effect is significantly dampened in cardiac-specific Nrf2 knockout mice [131]. Tan IIA can also upregulate AMPK expression and downregulate mammalian target of rapamycin (mTOR) expression to inhibit apoptosis [132]. Finally, Tan IIA can reduce apoptosis via inhibition of endoplasmic reticulum stress [146].

It was found that the apoptosis rate was reduced by AS-IV treatment which was associated with increasing Bcl2 levels, decreasing the expression of calpain-1 and improving the integrity of the mitochondrial structure and the mitochondrial membrane potential (MMP) in ISO-induced hypertrophic cardiomyocytes; moreover, AS-IV also increased the expression of mitochondrial superoxide dismutase (mito-SOD) [133]. Furthermore, AS-IV significantly inhibited apoptosis via the PI3K/Akt signaling pathway in DOX-induced cardiotoxicity [134]. Finally, AS-IV improved the cardiac function of HF mice by decreasing the levels of ROS and $\mathrm{H} 2 \mathrm{O} 2$ in the myocardium, suppressing the decrease in mitochondrial membrane potential and decreasing myocardial cell apoptosis by decreasing the expression of Bcl-2 and increasing the expression of cleaved caspase- 3 and Bax. These functions were mediated by inhibiting the increased expression of small ubiquitin-like modifier (SUMO)-specific protease 1 (Senp1) [135].

\section{Conclusions and perspectives}

As discussed above, HF is associated with multiple risk factors. Therefore, multitargeted treatments may be more effective in treating HF than therapy against a single target. However, chemical drugs do not have ideal therapeutic effects according to recent clinical studies. The combined application of herbal and chemical drugs for the treatment of HF is emerging as a trend in modern medicine. Natural products from Chinese herbs have been shown to have anticardiac hypertrophy, antifibrotic, antiinflammatory, antioxidative and antiapoptotic properties. However, there are several limitations to these findings. First, most of the evidence did not come from clinical studies, and there is a lack of large scale, multicenter, randomized and controlled clinical trials for the use of these drugs to treat HF. Second, it has been shown that at high concentrations, some natural products have toxic effects on embryonic development [147]. The systemic and organ-specific toxic effects and the minimally toxic dose of these natural products remain to be investigated. Finally, HF is caused by multiple factors. A large part of the published literature has focused on examining the effects of natural products on one or a few aspects of HF. Despite these limitations, further clinical trials and experimental studies will provide a better understanding of the mechanism of natural products from Chinese herbs to promote the development of these natural products from Chinese herbs for the treatment and prevention of $\mathrm{HF}$.

\section{Author contributions}

LHX, LYC and GYG wrote the manuscript with support from YW, YZX and YGZ; LHX LYC and GYG revised the manuscript under the guidance of YW, YZX and YGZ. All authors read and approved the final manuscript.

\section{Ethics approval and consent to participate}

Not applicable.

\section{Acknowledgment}

We would like to thank the key medical discipline (Cardiovascular medicine) of Hangzhou for supporting references material during the preparation of this manuscript.

\section{Funding}

This research was funded by the Science and Technology Program of Traditional Chinese Medicine in Zhejiang Province (2022ZZ028).

\section{Conflict of interest}

The authors declare no conflict of interest.

\section{References}

[1] Mentz RJ, O'Connor CM. Pathophysiology and clinical evaluation of acute heart failure. Nature Reviews Cardiology. 2016; 13: $28-35$.

[2] Ziaeian B, Fonarow GC. Epidemiology and aetiology of heart failure. Nature Reviews Cardiology. 2016; 13: 368-378.

[3] Wang H, Chai K, Du M, Wang S, Cai J, Li Y, et al. Prevalence and Incidence of Heart Failure among Urban Patients in China: A National Population-Based Analysis. Circulation: Heart Failure. 2021; 14: e008406. 
[4] Braunwald E. Heart failure. JACC. Heart Failure. 2013; 1: 1-20.

[5] Armstrong PW, Pieske B, Anstrom KJ, Ezekowitz J, Hernandez AF, Butler J, et al. Vericiguat in Patients with Heart Failure and Reduced Ejection Fraction. New England Journal of Medicine. 2020; 382: 1883-1893.

[6] Liu LCY, Schutte E, Gansevoort RT, van der Meer P, Voors AA. Finerenone: third-generation mineralocorticoid receptor antagonist for the treatment of heart failure and diabetic kidney disease. Expert Opinion on Investigational Drugs. 2015; 24: 1123-1135.

[7] Bakris GL, Agarwal R, Chan JC, Cooper ME, Gansevoort RT, Haller $\mathrm{H}$, et al. Effect of Finerenone on Albuminuria in Patients with Diabetic Nephropathy: A Randomized Clinical Trial. JAMA. 2015; 314: 884-894.

[8] Ponikowski P, Kirwan BA, Anker SD, McDonagh T, Dorobantu $\mathrm{M}$, Drozdz J, et al. Ferric carboxymaltose for iron deficiency at discharge after acute heart failure: a multicentre, double-blind, randomised, controlled trial. Lancet. 2020; 396: 1895-1904.

[9] Yancy CW, Jessup M, Bozkurt B, Butler J, Casey DE, Colvin MM, et al. 2016 ACC/AHA/HFSA Focused Update on New Pharmacological Therapy for Heart Failure: An Update of the 2013 ACCF/AHA Guideline for the Management of Heart Failure: a Report of the American College of Cardiology/American Heart Association Task Force on Clinical Practice Guidelines and the Heart Failure Society of America. Circulation. 2016; 134: e282-e293.

[10] Tsai M, Hu W, Lin C, Lee Y, Chen S, Hung Y, et al. Prescription pattern of Chinese herbal products for heart failure in Taiwan: a population-based study. International Journal of Cardiology. 2017; 228: 90-96.

[11] Tang WHW, Huang Y. Cardiotonic modulation in heart failure: insights from traditional Chinese medicine. Journal of the American College of Cardiology. 2013; 62: 1073-1074.

[12] McDonagh TA, Metra M, Adamo M, Gardner RS, Baumbach A, Bohm M, et al. $2021 \mathrm{ESC}$ Guidelines for the diagnosis and treatment of acute and chronic heart failure. European Heart Journal. 2021; 42: 3599-3726.

[13] Tanai E, Frantz S. Pathophysiology of Heart Failure. Comprehensive Physiology. 2015; 6: 187-214.

[14] Schirone L, Forte M, Palmerio S, Yee D, Nocella C, Angelini F, et al. A Review of the Molecular Mechanisms Underlying the Development and Progression of Cardiac Remodeling. Oxidative Medicine and Cellular Longevity. 2017; 2017: 3920195.

[15] Oka T, Akazawa H, Naito AT, Komuro I. Angiogenesis and cardiac hypertrophy: maintenance of cardiac function and causative roles in heart failure. Circulation Research. 2014; 114: 565-571.

[16] Tham YK, Bernardo BC, Ooi JYY, Weeks KL, McMullen JR. Pathophysiology of cardiac hypertrophy and heart failure: signaling pathways and novel therapeutic targets. Archives of Toxicology. 2015; 89: 1401-1438.

[17] Marks AR. Calcium cycling proteins and heart failure: mechanisms and therapeutics. The Journal of Clinical Investigation. 2013; 123: 46-52.

[18] Arai M, Alpert NR, MacLennan DH, Barton P, Periasamy M. Alterations in sarcoplasmic reticulum gene expression in human heart failure. a possible mechanism for alterations in systolic and diastolic properties of the failing myocardium. Circulation Research. 1993; 72: 463-469.

[19] Hasenfuss G, Reinecke H, Studer R, Meyer M, Pieske B, Holtz $\mathrm{J}$, et al. Relation between myocardial function and expression of sarcoplasmic reticulum $\mathrm{Ca}(2+)$-ATPase in failing and nonfailing human myocardium. Circulation Research. 1994; 75: 434-442.

[20] Marx SO, Reiken S, Hisamatsu Y, Jayaraman T, Burkhoff D, Rosemblit N, et al. PKA phosphorylation dissociates FKBP12.6 from the calcium release channel (ryanodine receptor): defective regulation in failing hearts. Cell. 2000; 101: 365-376.

[21] Ather S, Respress JL, Li N, Wehrens XHT. Alterations in ryan- odine receptors and related proteins in heart failure. Biochimica Et Biophysica Acta. 2013; 1832: 2425-2431.

[22] Zhang T, Maier LS, Dalton ND, Miyamoto S, Ross J, Bers DM, et al. The deltaC isoform of CaMKII is activated in cardiac hypertrophy and induces dilated cardiomyopathy and heart failure. Circulation Research. 2003; 92: 912-919.

[23] Grimm M, Ling H, Brown JH. Crossing signals: relationships between $\beta$-adrenergic stimulation and CaMKII activation. Heart Rhythm. 2011; 8: 1296-1298.

[24] Bond RC, Bryant SM, Watson JJ, Hancox JC, Orchard CH, James AF. Reduced density and altered regulation of rat atrial Ltype $\mathrm{Ca}(2+)$ current in heart failure. American Journal of Physiology. Heart and Circulatory Physiology. 2017; 312: H384H391.

[25] Hobai IA, O’Rourke B. Decreased sarcoplasmic reticulum calcium content is responsible for defective excitation-contraction coupling in canine heart failure. Circulation. 2001; 103: $1577-$ 1584 .

[26] McMullen JR, Jennings GL. Differences between pathological and physiological cardiac hypertrophy: novel therapeutic strategies to treat heart failure. Clinical and Experimental Pharmacology \& Physiology. 2007; 34: 255-262.

[27] Bernardo BC, Weeks KL, Pretorius L, McMullen JR. Molecular distinction between physiological and pathological cardiac hypertrophy: experimental findings and therapeutic strategies. Pharmacology \& Therapeutics. 2010; 128: 191-227.

[28] Shimoura H, Tanaka H, Matsumoto K, Mochizuki Y, Hatani $\mathrm{Y}$, Hatazawa K, et al. Effects of a changeover from other angiotensin II receptor blockers to olmesartan on left ventricular hypertrophy in heart failure patients. Heart and Vessels. 2017; 32: $584-590$

[29] Tsutsui H, Kinugawa S, Matsushima S. Oxidative stress and heart failure. American Journal of Physiology. Heart and Circulatory Physiology. 2011; 301: H2181-H2190.

[30] van der Pol A, van Gilst WH, Voors AA, van der Meer P. Treating oxidative stress in heart failure: past, present and future. European Journal of Heart Failure. 2019; 21: 425-435.

[31] Heymes C, Bendall JK, Ratajczak P, Cave AC, Samuel J, Hasenfuss $\mathrm{G}$, et al. Increased myocardial NADPH oxidase activity in human heart failure. Journal of the American College of Cardiology. 2003; 41: 2164-2171.

[32] Takimoto E, Kass DA. Role of oxidative stress in cardiac hypertrophy and remodeling. Hypertension. 2007; 49: 241-248.

[33] Berk BC, Fujiwara K, Lehoux S. ECM remodeling in hypertensive heart disease. Journal of Clinical Investigation. 2007; 117: 568-575.

[34] Oldershaw PJ, Brooksby IA, Davies MJ, Coltart DJ, Jenkins BS, Webb-Peploe MM. Correlations of fibrosis in endomyocardial biopsies from patients with aortic valve disease. British Heart Journal. 1980; 44: 609-611.

[35] Segura AM, Frazier OH, Buja LM. Fibrosis and heart failure. Heart Failure Reviews. 2014; 19: 173-185.

[36] Lakhani HV, Khanal T, Gabi A, Yousef G, Alam MB, Sharma $\mathrm{D}$, et al. Developing a panel of biomarkers and miRNA in patients with myocardial infarction for early intervention strategies of heart failure in West Virginian population. PLoS ONE. 2018; 13: e0205329.

[37] Desmoulière A, Geinoz A, Gabbiani F, Gabbiani G. Transforming growth factor-beta 1 induces alpha-smooth muscle actin expression in granulation tissue myofibroblasts and in quiescent and growing cultured fibroblasts. The Journal of Cell Biology. 1993; 122: 103-111.

[38] Kuwahara F, Kai H, Tokuda K, Kai M, Takeshita A, Egashira $\mathrm{K}$, et al. Transforming growth factor-beta function blocking prevents myocardial fibrosis and diastolic dysfunction in pressureoverloaded rats. Circulation. 2002; 106: 130-135. 
[39] Flanders KC. Smad3 as a mediator of the fibrotic response. International Journal of Experimental Pathology. 2004; 85: 47-64.

[40] Schiller M, Javelaud D, Mauviel A. TGF-beta-induced SMAD signaling and gene regulation: consequences for extracellular matrix remodeling and wound healing. Journal of Dermatological Science. 2004; 35: 83-92.

[41] von Haehling S, Schefold JC, Lainscak M, Doehner W, Anker SD. Inflammatory Biomarkers in Heart Failure Revisited: much more than Innocent Bystanders. Heart Failure Clinics. 2009; 5: 549-560.

[42] Braunwald E. Biomarkers in heart failure. The New England Journal of Medicine. 2008; 358: 2148-2159.

[43] Mann DL. Inflammatory mediators and the failing heart: past, present, and the foreseeable future. Circulation Research. 2002; 91: 988-998.

[44] Kacimi R, Vessey DA, Honbo N, Karliner JS. Adult cardiac fibroblasts null for sphingosine kinase-1 exhibit growth dysregulation and an enhanced proinflammatory response. Journal of Molecular and Cellular Cardiology. 2007; 43: 85-91.

[45] Bryant D, Becker L, Richardson J, Shelton J, Franco F, Peshock $\mathrm{R}$, et al. Cardiac Failure in Transgenic Mice with Myocardial Expression of Tumor Necrosis Factor- $\alpha$. Circulation. 1998; 97 : 1375-1381.

[46] Frantz S, Falcao-Pires I, Balligand J, Bauersachs J, Brutsaert D, Ciccarelli $\mathrm{M}$, et al. The innate immune system in chronic cardiomyopathy: A European Society of Cardiology (ESC) scientific statement from the Working Group on Myocardial Function of the ESC. European Journal of Heart Failure. 2018; 20: 445459 .

[47] Bartekova M, Radosinska J, Jelemensky M, Dhalla NS. Role of cytokines and inflammation in heart function during health and disease. Heart Failure Reviews. 2018; 23: 733-758.

[48] Blaser H, Dostert C, Mak TW, Brenner D. TNF and ROS Crosstalk in Inflammation. Trends in Cell Biology. 2016; 26: 249-261.

[49] Win S, Than TA, Fernandez-Checa JC, Kaplowitz N. JNK interaction with Sab mediates ER stress induced inhibition of mitochondrial respiration and cell death. Cell Death \& Disease. 2014; 5: e989.

[50] Everett BM, Cornel JH, Lainscak M, Anker SD, Abbate A, Thuren T, et al. Anti-Inflammatory Therapy with Canakinumab for the Prevention of Hospitalization for Heart Failure. Circulation. 2019; 139: 1289-1299.

[51] Adamo L, Rocha-Resende C, Prabhu SD, Mann DL. Reappraising the role of inflammation in heart failure. Nature Reviews Cardiology. 2020; 17: 269-285.

[52] Olivetti G, Abbi R, Quaini F, Kajstura J, Cheng W, Nitahara JA, et al. Apoptosis in the failing human heart. The New England Journal of Medicine. 1997; 336: 1131-1141.

[53] Corsetti G, Chen-Scarabelli C, Romano C, Pasini E, Dioguardi FS, Onorati F, et al. Autophagy and Oncosis/Necroptosis are Enhanced in Cardiomyocytes from Heart Failure Patients. Medical Science Monitor Basic Research. 2019; 25: 33-44.

[54] Abbate A, Bussani R, Amin MS, Vetrovec GW, Baldi A. Acute myocardial infarction and heart failure: role of apoptosis. The International Journal of Biochemistry \&Amp; Cell Biology. 2006; 38: 1834-1840.

[55] Balakumar P, Singh M. Anti-tumour necrosis factor-alpha therapy in heart failure: future directions. Basic \& Clinical Pharmacology \& Toxicology. 2006; 99: 391-397.

[56] Rodrigues V, Agrelli GS, Leon SC, Silva Teixeira DN, Tostes S, Rocha-Rodrigues DB. Fas/Fas-L expression, apoptosis and low proliferative response are associated with heart failure in patients with chronic Chagas' disease. Microbes and Infection. 2008; 10 : 29-37.

[57] Sayed D, He M, Hong C, Gao S, Rane S, Yang Z, et al.
MicroRNA-21 is a downstream effector of AKT that mediates its antiapoptotic effects via suppression of Fas ligand. The Journal of Biological Chemistry. 2010; 285: 20281-20290.

[58] Chung ES, Packer M, Lo KH, Fasanmade AA, Willerson JT. Randomized, double-blind, placebo-controlled, pilot trial of infliximab, a chimeric monoclonal antibody to tumor necrosis factor-alpha, in patients with moderate-to-severe heart failure: results of the anti-TNF Therapy against Congestive Heart Failure (ATTACH) trial. Circulation. 2003; 107: 3133-3140.

[59] Chaanine AH. Morphological Stages of Mitochondrial Vacuolar Degeneration in Phenylephrine-Stressed Cardiac Myocytes and in Animal Models and Human Heart Failure. Medicina. 2019; 55:239.

[60] Karch J, Kwong JQ, Burr AR, Sargent MA, Elrod JW, Peixoto $\mathrm{PM}$, et al. Bax and Bak function as the outer membrane component of the mitochondrial permeability pore in regulating necrotic cell death in mice. eLife. 2013; 2: e00772.

[61] Xu T, Ding W, Ao X, Chu X, Wan Q, Wang Y, et al. ARC regulates programmed necrosis and myocardial ischemia/reperfusion injury through the inhibition of mPTP opening. Redox Biology. 2019; 20: 414-426.

[62] Zhao C, Li S, Zhang J, Huang Y, Zhang L, Zhao F, et al. Current state and future perspective of cardiovascular medicines derived from natural products. Pharmacology \& Therapeutics. 2020; 216: 107698.

[63] Yu Y, Hu S, Li G, Xue J, Li Z, Liu X, et al. Comparative effectiveness of Di'ao Xin Xue Kang capsule and Compound Danshen tablet in patients with symptomatic chronic stable angina. Scientific Reports. 2014; 4: 7058.

[64] Jia Y, Chen C, Ng C, Leung S. Meta-Analysis of Randomized Controlled Trials on the Efficacy of Di'ao Xinxuekang Capsule and Isosorbide Dinitrate in Treating Angina Pectoris. EvidenceBased Complementary and Alternative Medicine. 2012; 2012: 904147.

[65] Shang H, Zhang J, Yao C, Liu B, Gao X, Ren M, et al. Qi-shenyi-qi dripping pills for the secondary prevention of myocardial infarction: a randomised clinical trial. Evidence-Based Complementary and Alternative Medicine. 2013; 2013: 738391.

[66] Li X, Zhang J, Huang J, Ma A, Yang J, Li W, et al. A Multicenter, Randomized, Double-Blind, Parallel-Group, PlaceboControlled Study of the Effects of Qili Qiangxin Capsules in Patients with Chronic Heart Failure. Journal of the American College of Cardiology. 2013; 62: 1065-1072.

[67] Zhou ZL, Yu P, Lin D. Study on effect of Astragalus injection in treating congestive heart failure. Chinese Journal of Integrated Traditional and Western Medicine. 2001; 21: 747-749.

[68] Liu Z, Xiong Z, Yu X. Effect of astragalus injection on immune function in patients with congestive heart failure. Chinese Journal of Integrated Traditional and Western Medicine. 2003; 23: 351-353.

[69] Xian S, Yang Z, Lee J, Jiang Z, Ye X, Luo L, et al. A randomized, double-blind, multicenter, placebo-controlled clinical study on the efficacy and safety of Shenmai injection in patients with chronic heart failure. Journal of Ethnopharmacology. 2016; 186: 136-142.

[70] Ma R, Wang C, Shen Y, Wang Z, Ma J, Huang L. Effect of Shenmai Injection on ventricular diastolic function in patients with chronic heart failure: an assessment by tissue Doppler imaging. Chinese Journal of Integrative Medicine. 2010; 16: 173-175.

[71] Wu H, Lu Y, Zhang Q. Effects of shenmai injection on plasma neuropeptide $\mathrm{Y}$, endothelin and atrial natriuretic polypeptide in heart failure patients. Chinese Journal of Integrated Traditional and Western Medicine. 1999; 19: 653-655.

[72] Hong Y, Xie W, Chen CS. Effect of shengmai injection on TRAIL death receptor of patients with congestive heart failure. Chinese Journal of Integrated Traditional and Western Medicine. 
2005; 25: 1092-1095.

[73] Ma L, Yang L, Chen TD. Influence of large amount of shengmai injection on blood coagulation in patients with chronic heart failure. Chinese Journal of Integrated Traditional and Western Medicine. 2003; 23: 275-277.

[74] Uretsky BF, Young JB, Shahidi FE, Yellen LG, Harrison MC, Jolly MK. Randomized study assessing the effect of digoxin withdrawal in patients with mild to moderate chronic congestive heart failure: results of the PROVED trial. PROVED Investigative Group. Journal of the American College of Cardiology. 1993; 22: 955-962.

[75] Bavendiek U, Berliner D, Dávila LA, Schwab J, Maier L, Philipp $\mathrm{SA}$, et al. Rationale and design of the DIGIT-HF trial (DIGitoxin to Improve ouTcomes in patients with advanced chronic Heart Failure): a randomized, double-blind, placebo-controlled study. European Journal of Heart Failure. 2019; 21: 676-684.

[76] Bussey HI, Hawkins DW, Gaspard JJ, Walsh RA. A Comparative Trial of Digoxin and Digitoxin in the Treatment of Congestive Heart Failure. Pharmacotherapy. 1988; 8: 235-240.

[77] Vercillo L, Rossi C, Vergallo L. On the use of desacetyllanatoside $\mathrm{C}$ in therapy of uncompensated heart diseases. Clinical contribution. Minerva Medica. 1965; 56: 3172-3175.

[78] Gábor G. Clinical and experimental experiences concerning the treatment of cardiac decompensation with lanatoside a digitalis glycoside. Therapia Hungarica (English Edition). 1967; 15: 113-116.

[79] Lu Y, Yan Y, Liu X. Effects of alprostadil combined with tanshinone IIa injection on microcirculation disorder, outcomes, and cardiac function in AMI patients after PCI. Annals of Palliative Medicine. 2021; 10: 97-103.

[80] Mao S, Taylor S, Chen Q, Zhang M, Hinek A. Sodium tanshinone IIA sulfonate prevents the adverse left ventricular remodelling: Focus on polymorphonuclear neutrophil-derived granule components. Journal of Cellular and Molecular Medicine. 2019; 23: 4592-4600.

[81] Fu S, Zhang J, Menniti-Ippolito F, Gao X, Galeotti F, Massari M, et al. Huangqi injection (a traditional Chinese patent medicine) for chronic heart failure: a systematic review. PLoS ONE. 2011; 6: e19604.

[82] Luo HM, Dai RH, Li Y. Nuclear cardiology study on effective ingredients of Astragalus membranaceus in treating heart failure. Chinese Journal of Integrated Traditional and Western Medicine. 1995; 15: 707-709.

[83] van Veldhuisen DJ, Man in 't Veld AJ, Dunselman PH, Lok DJ, Dohmen HJ, Poortermans JC, et al. Double-blind placebocontrolled study of ibopamine and digoxin in patients with mild to moderate heart failure: results of the Dutch Ibopamine Multicenter Trial (DIMT). Journal of the American College of Cardiology. 1993; 22: 1564-1573.

[84] Wang Y, Wang Q, Li C, Lu L, Zhang Q, Zhu R, et al. A Review of Chinese Herbal Medicine for the Treatment of Chronic Heart Failure. Current Pharmaceutical Design. 2017; 23: 5115-5124.

[85] Xie Z, Wang S, Liang Z, Zeng L. Effect of Zhenwu Tang Granule on pressure-overloaded left ventricular myocardial hypertrophy in rats. World Journal of Emergency Medicine. 2010; 1: 149153.

[86] Lai J, Wu Y, Hang L, Gael A, Deng T, Yan Q, et al. Zhenwu Decoction delays ventricular hypertrophy in rats with uremic cardiomyopathy. Journal of Southern Medical University. 2019; 39: 113-119.

[87] Mao HP, Wang XY, Gao YH, Chang YX, Chen L, Niu ZC, et al. Danhong injection attenuates isoproterenol-induced cardiac hypertrophy by regulating $\mathrm{p} 38$ and NF-kappab pathway. Journal of Ethnopharmacology. 2016; 186: 20-29.

[88] Li Y, Ruan X, Xu X, Li C, Qiang T, Zhou H, et al. Shengmai Injection Suppresses Angiotensin II-Induced Cardiomyocyte Hy- pertrophy and Apoptosis via Activation of the AMPK Signaling Pathway through Energy-Dependent Mechanisms. Frontiers in Pharmacology. 2019; 10: 1095.

[89] Picollo CT, Santos AAD, Antonio EL, Silva JMA, Bocalini D, Serra AJ, et al. Digitoxin Attenuates Heart Failure, Reduces Myocardial Hypertrophy, and Preserves the Calcium-Binding Proteins in Infarcted Rats. Journal of Cardiovascular Pharmacology and Therapeutics. 2020; 25: 265-272.

[90] Chan P, Liu I, Li Y, Yu W, Cheng J. Antihypertension Induced by Tanshinone IIA Isolated from the Roots of Salvia miltiorrhiza. Evidence-Based Complementary and Alternative Medicine. 2011; 2011: 392627.

[91] Jiang Q, Lu W, Yang K, Hadadi C, Fu X, Chen Y, et al. Sodium tanshinone IIA sulfonate inhibits hypoxia-induced enhancement of SOCE in pulmonary arterial smooth muscle cells via the PKGPPAR-gamma signaling axis. American Journal of Physiology. Cell Physiology. 2016; 311: C136-C149.

[92] Chen Y, Lee N, Pai P, Chung L, Shen C, Rajendran P, et al. Tanshinone-induced ERs suppresses IGFII activation to alleviate Ang II-mediated cardiac hypertrophy. Journal of Receptor and Signal Transduction Research. 2017; 37: 493-499.

[93] Ji Y, Wang T, Zhang X, Li L, Li L, Guo Y, et al. Astragalosides increase the cardiac diastolic function and regulate the "Calcium sensing receptor-protein kinase C-protein phosphatase 1" pathway in rats with heart failure. Biomedicine \& Pharmacotherapy. 2018; 103: 838-843.

[94] Liu Z, Liu H, Wang J. Astragaloside IV protects against the pathological cardiac hypertrophy in mice. Biomedicine \& Pharmacotherapy $=$ Biomedecine Pharmacotherapie. 2018; 97: $1468-1478$.

[95] Nie P, Meng F, Zhang J, Wei X, Shen C. Astragaloside IV Exerts a Myocardial Protective Effect against Cardiac Hypertrophy in Rats, Partially via Activating the Nrf2/HO-1 Signaling Pathway. Oxidative Medicine and Cellular Longevity. 2019; 2019: 4625912.

[96] Yang J, Wang HX, Zhang YJ, Yang YH, Lu ML, Zhang J, et al. Astragaloside IV attenuates inflammatory cytokines by inhibiting TLR4/NF-kB signaling pathway in isoproterenol-induced myocardial hypertrophy. Journal of Ethnopharmacology. 2013; 150: $1062-1070$.

[97] Pang L, Ju A, Zheng X, Li F, Song Y, Zhao Y, et al. YiQiFuMai Powder Injection attenuates coronary artery ligationinduced myocardial remodeling and heart failure through modulating MAPKs signaling pathway. Journal of Ethnopharmacology. 2017; 202: 67-77.

[98] Zhang Q, Guo D, Wang Y, Wang X, Wang Q, Wu Y, et al. Danqi Pill Protects Against Heart Failure Post-Acute Myocardial Infarction via HIF-1alpha/PGC-1alpha Mediated Glucose Metabolism Pathway. Frontiers in Pharmacology. 2020; 11: 458.

[99] Zhu J, Ye Q, Xu S, Chang Y, Liu X, Ma Y, et al. Shengmai injection alleviates $\mathrm{H} 2 \mathrm{O} 2$-induced oxidative stress through activation of AKT and inhibition of ERK pathways in neonatal rat cardiomyocytes. Journal of Ethnopharmacology. 2019; 239: 111677.

[100] Li Y, Elmer G, Leboeuf RC. Tanshinone IIA reduces macrophage death induced by hydrogen peroxide by upregulating glutathione peroxidase. Life Sciences. 2008; 83: 557-562.

[101] Yang G, Wang F, Wang Y, Yu X, Yang S, Xu H, et al. Protective effect of tanshinone IIA on H2O2-induced oxidative stress injury in rat cardiomyocytes by activating Nrf2 pathway. Journal of Receptors and Signal Transduction. 2020; 40: 264-272.

[102] Chen R, Chen W, Huang X, Rui Q. Tanshinone IIA attenuates heart failure via inhibiting oxidative stress in myocardial infarction rats. Molecular Medicine Reports. 2021; 23: 404.

[103] Li X, Ding Y, Wu H, Bian Z, Xu J, Gu C, et al. Astragaloside IV 
Prevents Cardiac Remodeling in the Apolipoprotein E-Deficient Mice by Regulating Cardiac Homeostasis and Oxidative Stress. Cellular Physiology and Biochemistry. 2017; 44: 2422-2438.

[104] Huang H, Lai S, Wan Q, Qi W, Liu J. Astragaloside IV protects cardiomyocytes from anoxia/reoxygenation injury by upregulating the expression of Hes1 protein. Canadian Journal of Physiology and Pharmacology. 2016; 94: 542-553.

[105] Dai H, Jia G, Lu M, Liang C, Wang Y, Wang H. Astragaloside IV inhibits isoprenaline-induced cardiac fibrosis by targeting the reactive oxygen species/mitogen-activated protein kinase signaling axis. Molecular Medicine Reports. 2017; 15: $1765-$ 1770 .

[106] Xu C, Tang F, Lu M, Yang J, Han R, Mei M, et al. Pretreatment with Astragaloside IV protects human umbilical vein endothelial cells from hydrogen peroxide induced oxidative stress and cell dysfunction via inhibiting eNOS uncoupling and NADPH oxidase-ROS-NF-kB pathway. Canadian Journal of Physiology and Pharmacology. 2016; 94: 1132-1140.

[107] Siman FDM, Silveira EA, Fernandes AA, Stefanon I, Vassallo DV, Padilha AS. Ouabain induces nitric oxide release by a PI3K/Akt-dependent pathway in isolated aortic rings from rats with heart failure. Journal of Cardiovascular Pharmacology. 2015; 65: 28-38.

[108] Chorvatova A, Elzwiei F, Mateasik A, Chorvat D. Effect of ouabain on metabolic oxidative state in living cardiomyocytes evaluated by time-resolved spectroscopy of endogenous NAD(P)H fluorescence. Journal of Biomedical Optics. 2012; 17: 101505.

[109] Yin Q, Lu H, Bai Y, Tian A, Yang Q, Wu J, et al. A metabolite of Danshen formulae attenuates cardiac fibrosis induced by isoprenaline, via a NOX2/ROS/p38 pathway. British Journal of Pharmacology. 2015; 172: 5573-5585.

[110] Ma J, Ma S, Yin C, Wu H. Shengmai San-derived herbal prevents the development of a vulnerable substrate for atrial fibrillation in a rat model of ischemic heart failure. Biomedicine \& Pharmacotherapy. 2018; 100: 156-167.

[111] Zhang G, Yang G, Deng Y, Zhao X, Yang Y, Rao J, et al. Ameliorative effects of Xue-Fu-Zhu-Yu decoction, Tian-Ma-GouTeng-Yin and Wen-Dan decoction on myocardial fibrosis in a hypertensive rat mode. BMC Complementary and Alternative Medicine. 2016; 16: 56.

[112] Wang J, Lu L, Wang Y, Wu Y, Han J, Wang W, et al. Qishenyiqi Dropping Pill attenuates myocardial fibrosis in rats by inhibiting RAAS-mediated arachidonic acid inflammation. Journal of Ethnopharmacology. 2015; 176: 375-384.

[113] Ma S, Li X, Dong L, Zhu J, Zhang H, Jia Y. Protective effect of Sheng-Mai Yin, a traditional Chinese preparation, against doxorubicin-induced cardiac toxicity in rats. BMC Complementary and Alternative Medicine. 2016; 16: 61.

[114] Chan P, Liu J, Lin L, Chen P, Cheng T, Lin J, et al. Tanshinone IIA inhibits angiotensin II-induced cell proliferation in rat cardiac fibroblasts. The American Journal of Chinese Medicine. 2011; 39: 381-394.

[115] Huang L, Zhu J, Zheng M, Zou R, Zhou Y, Zhu M. Tanshinone IIA protects against subclinical lipopolysaccharide induced cardiac fibrosis in mice through inhibition of NADPH oxidase. International Immunopharmacology. 2018; 60: 59-63.

[116] Lu J, Wang Q, Zhou Y, Lu X, Liu Y, Wu Y, et al. Astragaloside against cardiac fibrosis by inhibiting TRPM7 channel. Phytomedicine. 2017; 30: 10-17.

[117] Jia G, Leng B, Wang H, Dai H. Inhibition of cardiotrophin-1 overexpression is involved in the anti-fibrotic effect of Astrogaloside IV. Molecular Medicine Reports. 2017; 16: 8365-8370.

[118] Chang H, Wang Q, Shi T, Huo K, Li C, Zhang Q, et al. Effect of DanQi Pill on PPARalpha, lipid disorders and arachidonic acid pathway in rat model of coronary heart disease. BMC Comple- mentary and Alternative Medicine. 2016; 16: 103.

[119] Wang Y, Li C, Liu Z, Shi T, Wang Q, Li D, et al. DanQi Pill protects against heart failure through the arachidonic acid metabolism pathway by attenuating different cyclooxygenases and leukotrienes B4. BMC Complementary and Alternative Medicine. 2014; 14: 67.

[120] Chen J, Wei J, Orgah J, Zhu Y, Ni J, Li L, et al. Cardioprotective Effect of Danhong Injection against Myocardial Infarction in Rats is Critically Contributed by MicroRNAs. EvidenceBased Complementary and Alternative Medicine. 2019; 2019: 4538985.

[121] Gao S, Li L, Li L, Ni J, Guo R, Mao J, et al. Effects of the combination of tanshinone IIA and puerarin on cardiac function and inflammatory response in myocardial ischemia mice. Journal of Molecular and Cellular Cardiology. 2019; 137: 59-70.

[122] Feng J, Li S, Chen H. Tanshinone IIA inhibits myocardial remodeling induced by pressure overload via suppressing oxidative stress and inflammation: Possible role of silent information regulator 1. European Journal of Pharmacology. 2016; 791: 632-639.

[123] Cheng S, Yu P, Yang L, Shi H, He A, Chen H, et al. Astragaloside IV enhances cardioprotection of remote ischemic conditioning after acute myocardial infarction in rats. American Journal of Translational Research. 2016; 8: 4657-4669.

[124] Zhao P, Wang Y, Zeng S, Lu J, Jiang T, Li Y. Protective effect of astragaloside IV on lipopolysaccharide-induced cardiac dysfunction via downregulation of inflammatory signaling in mice. Immunopharmacology and Immunotoxicology. 2015; 37: 428433.

[125] Matsumori A, Ono K, Nishio R, Igata H, Shioi T, Matsui S, et al. Modulation of cytokine production and protection against lethal endotoxemia by the cardiac glycoside ouabain. Circulation. 1997; 96: 1501-1506.

[126] Zhong Y, Li M, Zhang X, Chen L, Wang Y, Xu Y. Dissecting Chemical Composition and Cardioprotective Effects of Fuzhengkangfu Decoction against Doxorubicin-Induced Cardiotoxicity by LC-MS and Bioinformatics Approaches. ACS Omega. 2020; 5: 14051-14060.

[127] Xiao J, Deng S, She Q, Li J, Kao G, Wang J, et al. Traditional Chinese medicine Qili qiangxin inhibits cardiomyocyte apoptosis in rats following myocardial infarction. Experimental and Therapeutic Medicine. 2015; 10: 1817-1823.

[128] Chen Y, Tang Y, Xiang Y, Xie Y, Huang X, Zhang Y. Shengmai injection improved doxorubicin-induced cardiomyopathy by alleviating myocardial endoplasmic reticulum stress and caspase-12 dependent apoptosis. BioMed Research International. 2015; 2015: 952671.

[129] Li X, Xiang D, Shu Y, Zeng X, Li Y. Mitigating effect of tanshinone IIA on ventricular remodeling in rats with pressure overload-induced heart failure. Acta Cirurgica Brasileira. 2019; 34: e201900807.

[130] Hong H, Liu J, Chen P, Chen J, Chan P, Cheng T. Tanshinone IIA prevents doxorubicin-induced cardiomyocyte apoptosis through Akt-dependent pathway. International Journal of Cardiology. 2012; 157: 174-179.

[131] Yan S, Zhao N, Geng Z, Shen J, Liu F, Yan D, et al. Modulations of Keap1-Nrf2 signaling axis by TIIA ameliorated the oxidative stress-induced myocardial apoptosis. Free Radical Biology \& Medicine. 2018; 115: 191-201.

[132] Zhang X, Wang Q, Wang X, Chen X, Shao M, Zhang Q, et al. Tanshinone IIA protects against heart failure post-myocardial infarction via AMPKs/mTOR-dependent autophagy pathway. Biomedicine \& Pharmacotherapy. 2019; 112: 108599.

[133] Mei M, Tang F, Lu M, He X, Wang H, Hou X, et al. Astragaloside IV attenuates apoptosis of hypertrophic cardiomyocyte through inhibiting oxidative stress and calpain-1 activation. En- 
vironmental Toxicology and Pharmacology. 2015; 40: 764-773.

[134] Jia Y, Zuo D, Li Z, Liu H, Dai Z, Cai J, et al. Astragaloside IV inhibits doxorubicin-induced cardiomyocyte apoptosis mediated by mitochondrial apoptotic pathway via activating the PI3K/Akt pathway. Chemical \& Pharmaceutical Bulletin. 2014; 62: 4553.

[135] Liu J, Li Y, Bian X, Xue N, Yu J, Dai S, et al. Astragaloside IV alleviates heart failure by regulating SUMO-specific protease 1 . Experimental and Therapeutic Medicine. 2021; 22: 1076.

[136] Zhang M, Guo F, Wu H, Yu Y, Wei J, Wang S, et al. DanHong injection targets endothelin receptor type $\mathrm{B}$ and angiotensin II receptor type 1 in protection against cardiac hypertrophy. Oncotarget. 2017; 8: 103393-103409.

[137] Chen X, Wu R, Kong Y, Yang Y, Gao Y, Sun D, et al. Tanshinone IIA attenuates renal damage in STZ-induced diabetic rats via inhibiting oxidative stress and inflammation. Oncotarget. 2017; 8: 31915-31922.

[138] Zhang X, He D, Xu L, Ling S. Protective effect of tanshinone IIA on rat kidneys during hypothermic preservation. Molecular Medicine Reports. 2012; 5: 405-409.

[139] Wlodarczyk M, Glensk M. An in-depth look into a well-known herbal drug: Fingerprinting, isolation, identification, and content estimation of saponins in different Strophanthus seeds. Planta Medica. 2021. (in press)

[140] Li Y, Liu Y, Hu B, Chang X, Fan J, Sun K, et al. Attenuating effect of post-treatment with QiShen YiQi Pills on myocardial fibrosis in rat cardiac hypertrophy. Clinical Hemorheology and Microcirculation. 2012; 51: 177-191.
[141] Bi Z, Wang Y, Zhang W. A comprehensive review of tanshinone IIA and its derivatives in fibrosis treatment. Biomedicine \& Pharmacotherapy. 2021; 137: 111404.

[142] Lv Y, Pan Y, Gao Y, Lu J, Li Y, Bai J, et al. Effect of Danhong Injection Combined with Naoxintong Tablets on Prognosis and Inflammatory Factor Expression in Acute Coronary Syndrome Patients Undergoing Percutaneous Coronary Intervention. Acta Cardiologica Sinica. 2015; 31: 301-307.

[143] Qin X, Li T, Yan L, Liu Q, Tian Y. Tanshinone IIA protects against immune-mediated liver injury through activation of tcell subsets and regulation of cytokines. Immunopharmacology and Immunotoxicology. 2010; 32: 51-55.

[144] Kobayashi M, Usui-Kawanishi F, Karasawa T, Kimura H, Watanabe S, Mise N, et al. The cardiac glycoside ouabain activates NLRP3 inflammasomes and promotes cardiac inflammation and dysfunction. PLoS ONE. 2017; 12: e0176676.

[145] Sun J, Qian H, Li X, Tang X. QishenYiqi Dripping Pill Improves Heart Failure by Up-Regulation of $\beta 2$-Adrenergic Receptor Expression. The Journal of Heart Valve Disease. 2017; 26: 193-199.

[146] Chen J, Bi Y, Chen L, Zhang Q, Xu L. Tanshinone IIA exerts neuroprotective effects on hippocampus-dependent cognitive impairments in diabetic rats by attenuating ER stress-induced apoptosis. Biomedicine \& Pharmacotherapy. 2018; 104: 530536.

[147] Mossa AH, Mohafrash SMM, Chandrasekaran N. Safety of Natural Insecticides: Toxic Effects on Experimental Animals. BioMed Research International. 2018; 2018: 4308054. 